\title{
Minimal fundamental partial compositeness
}

\author{
Giacomo Cacciapaglia, ${ }^{1, *}$ Helene Gertov, ${ }^{2, \dagger}$ Francesco Sannino, ${ }^{2,3,4, \$}$ and Anders Eller Thomsen ${ }^{2, \S}$ \\ ${ }^{1}$ Univ Lyon, Université Lyon 1, CNRS/IN2P3, IPNL, F-69622 Villeurbanne, France \\ ${ }^{2} \mathrm{CP}^{3}$-Origins, University of Southern Denmark, Campusvej 55, 5230 Odense, Denmark \\ ${ }^{3}$ Danish IAS, University of Southern Denmark, 5230 Odense, Denmark \\ ${ }^{4}$ Theoretical Physics Department, CERN, 1211 Geneva 23, Switzerland
}

(Received 20 July 2017; revised manuscript received 1 May 2018; published 5 July 2018)

\begin{abstract}
Building upon the fundamental partial compositeness framework, we provide consistent and potentially complete composite extensions of the Standard Model. These are used to determine the effective operators emerging at the electroweak scale in terms of the standard model fields upon consistently integrating out the heavy composite dynamics. We exhibit the first effective field theories matching these composite theories of flavor and analyze their physical consequences for the third generation quarks. Relations with other approaches, ranging from effective analyses for partial compositeness to extra dimensions as well as purely fermionic extensions, are briefly discussed. Our methodology is applicable to any composite theory of dynamical electroweak symmetry breaking featuring a complete theory of flavor.
\end{abstract}

DOI: 10.1103/PhysRevD.98.015006

\section{INTRODUCING THE MINIMAL FUNDAMENTAL COMPOSITE MODEL}

Since the earliest proposals of new composite dynamics (aka Technicolor-TC) as the underlying theory of electroweak symmetry breaking $[1,2]$, generating masses for the Standard Model (SM) fermions has been the biggest hurdle on the way to a complete model. Many attempts have been made, from extending the TC gauge sector [3] to introducing scalar mediators as in bosonic TC [4-9]. The SM fermion masses are generated either by effective operators bilinear in the fermion spinors, or via linear mixing to a fermionic bound state as in the partial compositeness mechanism [10]. In all cases, the main difficulty has been to construct a complete theory in the ultraviolet (UV). Phenomenologically it is difficult to accommodate a heavy top quark with the stringent bounds on the scale of flavor violation in the light quark and lepton sectors. Recently, in Ref. [11], an alternative paradigm has been introduced that allows for writing a potentially complete UV theory of composite flavor. The models account for a pseudo Nambu Goldstone boson (pNGB) Higgs particle [12] and can possibly be extrapolated to the strong gravity scale. Here

\footnotetext{
g.cacciapaglia@ipnl.in2p3.fr gertov@cp3.sdu.dk sannino@cp3.dias.sdu.dk

§aethomsen@cp3.sdu.dk
}

Published by the American Physical Society under the terms of the Creative Commons Attribution 4.0 International license. Further distribution of this work must maintain attribution to the author(s) and the published article's title, journal citation, and DOI. Funded by SCOAP. fermion masses are generated via Yukawa couplings involving TC-charged scalars. Partial compositeness is thus obtained at low energy by the formation of fermion-scalar bound states. For the top quark, the values of these Yukawas are required to be large at the condensation scale; thus further investigation is needed to prove that their running can be extrapolated all the way to the Planck scale without further new physics. This is based on an order of magnitude estimate; thus favorable strong form factors may avoid this potential issue. Composite theories including (super) TC scalars, attempting to give masses to some of the SM fermions, appeared earlier in the literature [13-18] for (walking) TC theories that did not feature a pseudo Nambu Goldstone boson Higgs particle.

In models of fundamental partial compositeness (FPC) the SM is extended with a new TC sector featuring new elementary fermions and scalars charged under a new gauge group $G_{\mathrm{TC}}$ [11]. Electroweak symmetry breaking (EWSB) is caused by the TC dynamics in which the Higgs boson is replaced by a light composite state.

The TC Lagrangian before introducing the electroweak sector reads

$$
\begin{aligned}
\mathcal{L}_{\mathrm{TC}}= & -\frac{1}{4} \mathcal{G}_{\mu \nu} \mathcal{G}^{\mu \nu}+i \overline{\mathcal{F}} \bar{\sigma}^{\mu} D_{\mu} \mathcal{F}-\left(\frac{1}{2} \mathcal{F} m_{\mathcal{F}} \epsilon_{\mathrm{TC}} \mathcal{F}+\text { H.c. }\right) \\
& +\left(D_{\mu} \mathcal{S}\right)^{\dagger}\left(D^{\mu} \mathcal{S}\right)-\mathcal{S}^{\dagger} m_{\mathcal{S}}^{2} \mathcal{S}-V(\mathcal{S}),
\end{aligned}
$$

where TC-fermions and TC-scalars are in pseudoreal representations of the $G_{\mathrm{TC}}$ group, $m_{\mathcal{F}}$ and $m_{\mathcal{S}}^{2}$ are mass matrices and $\epsilon_{\mathrm{TC}}$ is the antisymmetric invariant tensor of $G_{\mathrm{TC}}$. This choice of representation is due to the fact that the most minimal models are of this nature [19]. Nevertheless 
the following analysis and methodology is generalisable to complex and real representations as well, for which a list of FPC models was made in [11].

Assuming $N_{\mathcal{F}}$ Weyl TC-fermions the maximal quantum global symmetry of the fermions in the kinetic term is $\mathrm{SU}\left(N_{\mathcal{F}}\right)$. The symmetries are such that $m_{\mathcal{F}}$ is an antisymmetric tensor in flavor space.

As the TC-scalars transform according to the same representation as the TC-fermions with respect to the new gauge group, no Yukawa interactions among the TCfermions and TC-scalars can be written (except for a few exceptions [11]). This implies that, with zero mass terms, the TC-scalars have an independent $\operatorname{Sp}\left(2 N_{\mathcal{S}}\right)$ symmetry. We assume the potential $V(\mathcal{S})$ to respect the maximum global symmetries of the TC theory. To elucidate the symmetry in the scalar sector we note that the $N_{\mathcal{S}}$ complex TC-scalars can be arranged in the following single field:

$$
\Phi=\left(\begin{array}{c}
\mathcal{S} \\
-\epsilon_{\mathrm{TC}} \mathcal{S}^{*}
\end{array}\right)
$$

still transforming according to a pseudoreal representation of $G_{\mathrm{TC}}$. The TC indices are hidden to keep the notation light, cf. Appendix A. One can show that this rearrangement leaves the TC Lagrangian invariant under the $\operatorname{Sp}\left(2 N_{\mathcal{S}}\right)$ flavor symmetry. The scalar kinetic and mass term now reads

$$
\frac{1}{2}\left(D_{\mu} \Phi\right) \epsilon_{\mathrm{TC}} \epsilon\left(D^{\mu} \Phi\right)-\frac{1}{2} \Phi \epsilon_{\mathrm{TC}} M_{\mathcal{S}}^{2} \Phi
$$

with

$$
M_{\mathcal{S}}^{2}=\left(\begin{array}{cc}
0 & -m_{\mathcal{S}}^{2 T} \\
m_{\mathcal{S}}^{2} & 0
\end{array}\right),
$$

and $\epsilon$ is the invariant symplectic form of $\operatorname{Sp}\left(2 N_{\mathcal{S}}\right)$.

A straightforward realization for this model is obtained choosing $G_{\mathrm{TC}}=\operatorname{Sp}(2 N)$ with the TC fundamental states in the fundamental representation. In Table I we summarize the elementary states of the TC theory as well as the bilinear gauge singlets along with their global transformation properties and multiplicities.

When adding the electroweak (EW) sector we embed it within the $\mathrm{SU}\left(N_{\mathcal{F}}\right)$ of the TC-fermion sector. In this way the EWSB is tied to the breaking of $\mathrm{SU}\left(N_{\mathcal{F}}\right)$ and the Higgs boson can be identified with a pNGB of the theory $[12,19]$. Assuming for the scalars a positive mass squared, it is natural to expect spontaneous symmetry breaking in the fermion sector $^{1}$ according to the pattern $\mathrm{SU}\left(N_{\mathcal{F}}\right) \rightarrow \operatorname{Sp}\left(N_{\mathcal{F}}\right)$. This breaking pattern was established in the absence of scalars for $N_{\mathcal{F}}=4$ and $G_{\mathrm{TC}}=\operatorname{Sp}(2)$ via first principle lattice

\footnotetext{
${ }^{1}$ In [11] there is also a preliminary analysis of the potential conformal window including light TC-scalars that allows us to argue that the model is expected to be in a chirally broken phase.
}

TABLE I. The fundamental matter fields of the theory appear in the first two lines of the table, both transforming according to the fundamental representation of TC. The last three lines correspond to the bilinear composite TC singlet states. The number of states counts the Weyl fermions or real scalars.

\begin{tabular}{lccc}
\hline \hline States & $\mathrm{SU}\left(N_{\mathcal{F}}\right)$ & $\mathrm{Sp}\left(2 N_{\mathcal{S}}\right)$ & Number of states \\
\hline $\mathcal{F}$ & $\square$ & 1 & $2 N \times N_{\mathcal{F}}$ \\
$\Phi$ & 1 & $\square$ & $2 N \times 2 N_{\mathcal{S}}$ \\
$\Phi \Phi$ & 1 & $1+\square$ & $1+N_{\mathcal{S}}\left(2 N_{\mathcal{S}}-1\right)$ \\
$\mathcal{F} \Phi$ & $\square$ & $\square$ & $2 N_{\mathcal{S}} N_{\mathcal{F}}$ \\
$\mathcal{F} \mathcal{F}$ & $\square$ & 1 & $N_{\mathcal{F}}\left(N_{\mathcal{F}}-1\right)$ \\
\hline \hline
\end{tabular}

simulations [20]. The ensuing TC-fermion bilinear condensate is

$$
\left\langle\mathcal{F}^{a} \epsilon_{\mathrm{TC}} \mathcal{F}^{a^{\prime}}\right\rangle=f_{\mathrm{TC}}^{2} \Lambda_{\mathrm{TC}} \Sigma_{0}^{a a^{\prime}}
$$

where Lorentz and TC indices are opportunely contracted, and the $\Sigma_{0}$ matrix is an antisymmetric, two-index representation of $\mathrm{SU}\left(N_{\mathcal{F}}\right)$. We also have $\Lambda_{\mathrm{TC}}=4 \pi f_{\mathrm{TC}}$ with $\Lambda_{\mathrm{TC}}$ the composite scale of the theory and $f_{\mathrm{TC}}$ the associated pion decay constant.

In addition, we envision two possibilities for the TCscalars: the formation of a condensate $\left\langle\Phi^{i} \epsilon_{\mathrm{TC}} \Phi^{j}\right\rangle$ may not happen or be proportional to the singlet of $\operatorname{Sp}\left(2 N_{\mathcal{S}}\right)$, in which case the flavor symmetry in the scalar sector is left unbroken, or a condensate forms and breaks $\operatorname{Sp}\left(2 N_{\mathcal{S}}\right)$ generating light bosonic degrees of freedom. For the remainder of this paper we will focus on the former case for the sake of simplicity.

We now turn our attention to the SM fermion mass generation. The presence of TC-scalars in FPC models allow for a new type of Yukawa interactions interfacing the TC and the SM sectors. In fact each new Yukawa operator involves a TC-fermion, a TC-scalar and a SM fermion and the new fundamental Yukawa Lagrangian to replace the SM one reads

$$
\mathcal{L}_{\text {yuk }}=-\psi^{i}{ }_{a} \epsilon_{i j} \Phi^{j} \epsilon_{\mathrm{TC}} \mathcal{F}^{a}+\text { H.c. }
$$

in which we make use of the spurion $\psi$ transforming under the relevant global symmetries as

$$
\psi^{i}{ }_{a} \equiv(\Psi y)^{i}{ }_{a} \in \square_{\mathcal{S}} \otimes \bar{\square}_{\mathcal{F}} .
$$

Here $\Psi$ is a generic SM fermion and $y$ is the new Yukawa matrix. With this spurionic construction we may formally consider $\mathcal{L}_{\text {yuk }}$ an invariant of the global TC symmetries. Additionally, the notation has the benefit that all Yukawa interactions are summarized in a single operator. Note that with the notation introduced here, the generation, color, and electroweak indices are all embedded in the global symmetries. At low energy, the Yukawa couplings in Eq. (6) 
generate linear mixing of the SM fermions with spin- $1 / 2$ resonances made of one TC-fermion and one TC-scalar (see Table I), thus implementing partial compositeness. This way of endowing masses for the SM fermions is free from long-standing problems in models of composite Higgs dynamics and, as we shall discuss later, can be also related to previous incomplete extensions.

Besides the SM fermions and Yukawas, the underlying theory contains two more spurions that explicitly break the flavor symmetries, that is the masses of the TC-fermions and scalars,

$$
m_{\mathcal{F}} \in \bar{\square}_{\mathcal{F}} \otimes 1_{\mathcal{S}}, \quad M_{\mathcal{S}}^{2} \in 1_{\mathcal{F}} \otimes \bar{\square}_{\mathcal{S}} .
$$

As they are dimensionful parameters, they can be inserted at the effective Lagrangian level only if an order parameter can be defined, i.e., either if the mass is small compared to the TC scale $\Lambda_{\mathrm{TC}}$, or if they are much larger. In the latter case, one can then expand in powers of the inverse of the mass matrices. We will start with the former case and classify the relevant operators in terms of powers of the spurion $\psi^{i}{ }_{a}$ and then discuss how to consistently move to the limit of large TC-scalar masses.

We are now ready to determine the effective operators emerging at the EW scale in terms of the SM fields upon consistently integrating out the heavy TC dynamics aside from the pNGB excitations. De facto we provide the first effective field theory that matches to a concrete and complete example of a composite theory of flavor. In turn, this allows for investigating its impact on electroweak observables and low energy flavor physics.

We structure the work as follows. In Sec. II we construct the effective field theory. We set the stage by first briefly reviewing the essentials of the TC pNGB effective field theory. We then move on to construct the symmetry allowed TC-induced effective operators involving SM fermions. We construct both fermion bilinears and fourfermions operators. Then we formulate the standard model induced one-loop pNGB potential and higher derivatives pNGB operators. Physical consequences and phenomenological constraints deriving from the third generation quarks physics are investigated in Sec. III, in which we also briefly comment on the light generations. Section IV is devoted to the relation with other approaches ranging from effective analyses for partial compositeness to extra dimensions as well as purely fermionic extensions. We finally offer our conclusions in Sec. V.

\section{EFFECTIVE FIELD THEORY AT THE ELECTROWEAK SCALE}

Having spelled out the underlying fundamental dynamics we now move to determine the effective operators at the EW scale. We start with a brief summary of the chiral Lagrangian for the TC sector. We then list the effective operators in terms of the SM fields generated by explicit realizations of partial compositeness. This is achieved by coherently matching the operators to the underlying composite flavor dynamics. This allows us, for the first time, to build in a controlled manner the full effective field theory. All operators will then appear in the Lagrangian

$$
\mathcal{L}_{\mathrm{EFT}}=\sum_{A} C_{A} \mathcal{O}_{A}+\left(\sum_{A} C_{A}^{\prime} \mathcal{O}_{A}^{\prime}+\text { H.c. }\right)
$$

for the effective field theory with coefficients $C_{A}^{(\prime)}$ determined by the underlying TC dynamics. Here $\mathcal{O}_{A}^{(\prime)}$ refers to the self-hermitian/complex operators respectively.

To organize the expansion of the EFT we adopt the counting of chiral dimension [21] as a generalization of the naive dimensional analysis (NDA) [22] for EW effective field theories with strong underlying dynamics. It will be apparent that this counting agrees with the naive estimates for the effective operators considered in Ref. [11]. In a realistic FPC model the power-counting is complicated slightly, by the potential occurrence of strong Yukawa couplings; achieving the correct top mass requires the product $y_{Q_{3}} y_{t} \sim 4 \pi$. Strong couplings in the chiral expansion, can potentially enhance certain operators beyond the order ascribed to them by simple counting of the chiral dimension. To alleviate this issue we defined the effective Yukawa couplings

$$
\frac{y_{\text {fund }}}{\sqrt{4 \pi}} \rightarrow y
$$

which are simple rescalings of the fundamental couplings. This will allow us to treat the Yukawa couplings as perturbative, albeit with a chiral dimension lowered to $1 / 2$ down from 1 . This is the prescription used in the remainder of this article. In the end, one must remember that the Yukawa parameters entering in the EFT are different from the fundamental Yukawa couplings by a rescaling.

For the underlying model to be fundamental, it must be possible to run a perturbative Yukawa coupling from the scale of strong gravity down to the scale of compositeness where it should become strong. The leading order beta function for the fundamental Yukawa coupling, $y_{t}$, belonging to the right-handed top quark (cf. Sec. III) in the presence of an $\operatorname{Sp}(2 N)$ technicolor group is ${ }^{2}$

$$
\frac{\partial y_{t}}{\partial \ln \mu}=\frac{y_{t}}{(4 \pi)^{2}} \frac{(4 N+10) y_{t}^{2}-(6 N+3) g_{\mathrm{TC}}^{2}}{4}
$$

in the absence of other Yukawa couplings. Starting the RG flow in the perturbative regime at high scales (e.g., the Planck scale) and evolving the couplings down to $\Lambda_{\mathrm{TC}}$, one

\footnotetext{
${ }^{2}$ This result differs from the analogous Eq. (32) in Ref. [11]; however the qualitative features of the running are retained.
} 
will find that $y_{t}$ increases in the IR as long as $g_{\mathrm{TC}} \geq$ $\sqrt{(4 N+10) /(6 N+3)} y_{t}$. With $g_{\mathrm{TC}}$ becoming strong at the scale of compositeness, we can expect that it pulls the Yukawa couplings with it. This effect, however, is outside the reach of perturbation theory and cannot be probed quantitatively, as one can only achieve $y_{t} \sim 1$, before $g_{\mathrm{TC}}$ becomes nonperturbative. A nonperturbative study is therefore required to quantitatively determine whether the requirement of a strong $y_{t}$ is compatible with completeness of the underlying model. This tension in the top Yukawas might also be resolved by favorable strong coefficients departing from unity by $O$ (few).

\section{A. Chiral Lagrangian setup}

The effective low-energy limit of the model may be described by a nonlinearly realized chiral Lagrangian, incorporating the Goldstone modes of the spontaneously broken symmetry $[23,24]$. As discussed in the previous section, the TC sector is invariant under the $\mathrm{SU}\left(N_{\mathcal{F}}\right)$ flavor symmetry, which is broken to the stability group $\operatorname{Sp}\left(N_{\mathcal{F}}\right)$ by the fermion condensate $\Sigma_{0}$. The breaking pattern will result in $N_{\mathcal{F}}\left(N_{\mathcal{F}}-1\right) / 2-1$ broken generators $X^{i}$ with corresponding (p)NGBs $\Pi_{i}$. The associated manifold $\mathrm{SU}\left(N_{\mathcal{F}}\right) / \mathrm{Sp}\left(N_{\mathcal{F}}\right)$ is parametrized by

$$
u(x)=\exp \left[\frac{\sqrt{2} i}{f_{\mathrm{TC}}} \Pi_{i}(x) X^{i}\right],
$$

having normalized the generators as $\operatorname{Tr}\left[X^{i} X^{j}\right]=\frac{1}{2} \delta^{i j}$. The Goldstone matrix $u$ transforms as

$$
u \rightarrow g u h^{\dagger},
$$

under flavor transformations, with $g \in \mathrm{SU}\left(N_{\mathcal{F}}\right)$. Here $h(g, \Pi) \in \operatorname{Sp}\left(N_{\mathcal{F}}\right)$ is a space-time dependent element of the stability group uniquely determined via the constraint $g u h^{\dagger} \in \mathrm{SU}\left(N_{\mathcal{F}}\right) / \operatorname{Sp}\left(N_{\mathcal{F}}\right)$. This results in a well-defined, though highly nontrivial transformation of the NGBs. Utilizing the fact that the broken generators satisfy $X_{i} \Sigma_{0}=$ $\Sigma_{0} X_{i}^{\mathrm{T}}$, one may parametrize the low-lying, pNGB, bilinear fermion composite states as

$$
\Sigma=u \Sigma_{0} u^{\mathrm{T}}=u^{2} \Sigma_{0}
$$

transforming like $\Sigma \rightarrow g \Sigma g^{\mathrm{T}}$ while leaving the vacuum alignment unchanged. This parametrization of the pNGBs around the vacuum coincides with that of Ref. [19] (even though the normalization of the decay constant is different).

As discussed in the previous section the SM gauge symmetries are embedded into the global symmetries. Parts of these are therefore promoted to local symmetries leading to the introduction of the covariant derivative $D_{\mu}$. With this gauging, the lowest order effective theory reads

$$
\mathcal{L}_{2}=\frac{1}{8} f_{\mathrm{TC}}^{2} \operatorname{Tr}\left[u_{\mu} u^{\mu}+\chi_{+}\right]
$$

Following Ref. [25] we introduced

$$
\begin{aligned}
& u_{\mu}=4 i X^{i} \operatorname{Tr}\left[X^{i} u^{\dagger} D_{\mu} u\right] \rightarrow h u_{\mu} h^{\dagger}, \\
& \chi_{ \pm}=u^{\dagger} \chi \Sigma_{0} u^{\dagger} \pm u \Sigma_{0} \chi^{\dagger} u \rightarrow h \chi_{ \pm} h^{\dagger},
\end{aligned}
$$

both transforming homogeneously under the stability group. The TC-fermion mass is encoded in $\chi=2 B_{0} m_{\mathcal{F}}^{*}$, where $B_{0}$ is a TC constant. Formally this is considered to be a spurion field which transforms as $\chi \rightarrow g \chi g^{\mathrm{T}}$ to preserve $\mathrm{SU}\left(N_{\mathcal{F}}\right)$ invariance through all steps. For a detailed discussion of the next-to-leading order (NLO) pion Lagrangian we refer to Refs. [26,27]. We also note that the chiral Lagrangian allows for the inclusion of a topological term, known as the WessZumino-Witten term, which has been gauged in [28].

\section{B. Effective bilinear operators with Standard Model fermions}

We now turn to the effective operators in terms of the SM fermion fields starting with the bilinear ones. They can be neatly organized according to their chiral dimension, starting with the lowest one which reads

$$
\mathcal{O}_{\text {Yuk }}=-\frac{f_{\mathrm{TC}}}{2}\left(\psi^{i_{1}}{ }_{a_{1}} \psi^{i_{2}} a_{2}\right) \Sigma^{a_{1} a_{2}} \epsilon_{i_{1} i_{2}} .
$$

The above corresponds to ordinary mass terms for the SM fermions and contains the Higgs couplings at linear order in the pNGB fields. The antisymmetric matrix $\epsilon_{i_{1} i_{2}}$, defined in Appendix A, contracts the $\operatorname{Sp}\left(2 N_{\mathcal{S}}\right)$ indices, while spinor indices are hidden with the convention that two Weyl spinors in parenthesis are contracted to a scalar.

At the next order we have the operator,

$$
\mathcal{O}_{\Pi f}=\frac{i f_{\mathrm{TC}}}{2 \Lambda_{\mathrm{TC}}}\left(\bar{\psi}^{i_{1} a_{1}} \bar{\sigma}_{\mu} \psi^{i_{2}} a_{2}\right) \Sigma_{a_{1} a_{3}}^{\dagger} \stackrel{\leftrightarrow}{D} \Sigma^{a_{3} a_{2}} \epsilon_{i_{1} i_{2}} .
$$

The above affects the coupling of massive gauge bosons, contained in the covariant derivative, to the SM fermions.

At next order again we find the dipole operators,

$\mathcal{O}_{f W}=\frac{f_{\mathrm{TC}}}{2 \Lambda_{\mathrm{TC}}^{2}}\left(\psi^{i_{1}}{ }_{a_{1}} \sigma^{\mu \nu} \psi^{i_{2}} a_{2}\right) A_{\mu \nu}^{I}\left(T_{\mathcal{F}}^{I} \Sigma-\Sigma\left(T_{\mathcal{F}}^{I}\right)^{\mathrm{T}}\right)^{a_{1} a_{2}} \epsilon_{i_{1} i_{2}}$,

$\mathcal{O}_{f G}=\frac{f_{\mathrm{TC}}}{2 \Lambda_{\mathrm{TC}}^{2}}\left(\psi^{i_{1}} a_{1} \sigma^{\mu \nu} \psi^{i_{2}} a_{2}\right) G_{\mu \nu}^{A} \Sigma^{a_{1} a_{2}}\left(\epsilon T_{\mathcal{S}}^{A}-\left(T_{\mathcal{S}}^{A}\right)^{\mathrm{T}} \epsilon\right)_{i_{1} i_{2}}$,

where $T_{\mathcal{F} / \mathcal{S}}^{k}$ are the generators of $\mathrm{SU}\left(N_{\mathcal{F}}\right)$ and $\operatorname{Sp}\left(2 N_{\mathcal{S}}\right)$ respectively, and $A_{\mu \nu}^{k} / G_{\mu \nu}^{k}$ the field strength tensors of the relative gauge bosons (more precisely, of the gauged subgroup). We note that the gauge couplings constants have been absorbed into the generators $T_{\mathcal{F} / \mathcal{S}}^{k}$ to account for there 
being several SM gauge groups embedded into each of them. The two operators, (20) and (21), have structures mimicking the Penguin-induced operators in the $\mathrm{SM}^{3}$

\section{Effective four-fermion operators with Standard Model fermions}

We now construct a consistent basis of four-fermion operators starting with five independent operators featuring two left-handed spinors $\psi$ and two right-handed ones $\bar{\psi}$,

$$
\begin{aligned}
\mathcal{O}_{4 f}^{1}= & \frac{1}{4 \Lambda_{\mathrm{TC}}^{2}}\left(\psi^{i_{1}}{ }_{a_{1}} \psi^{i_{2}}{ }_{a_{2}}\right)\left(\bar{\psi}^{i_{3} a_{3}} \bar{\psi}^{i_{4} a_{4}}\right) \\
& \times \Sigma^{a_{1} a_{2} \sum_{a_{3}}^{\dagger}} \epsilon_{a_{4}} \epsilon_{i_{1} i_{2}} \epsilon_{i_{3} i_{4}}, \\
\mathcal{O}_{4 f}^{2}= & \frac{1}{4 \Lambda_{\mathrm{TC}}^{2}}\left(\psi^{i_{1}}{ }_{a_{1}} \psi^{i_{2}}{ }_{a_{2}}\right)\left(\bar{\psi}^{i_{3} a_{3}} \bar{\psi}^{i_{4} a_{4}}\right) \\
& \times\left(\delta^{a_{1}}{ }_{a_{3}} \delta^{a_{2}} a_{a_{4}}-\delta^{a_{1}}{ }_{a_{4}} \delta^{a_{2}}{ }_{a_{3}}\right) \epsilon_{i_{1} i_{2}} \epsilon_{i_{3} i_{4}}, \\
\mathcal{O}_{4 f}^{3}= & \frac{1}{4 \Lambda_{\mathrm{TC}}^{2}}\left(\psi^{i_{1}}{ }_{a_{1}} \psi^{i_{2}}{ }_{a_{2}}\right)\left(\bar{\psi}^{i_{3} a_{3}} \bar{\psi}^{i_{4} a_{4}}\right) \\
& \left.\times \Sigma^{a_{1} a_{2}} \sum_{a_{3} a_{4}}^{\dagger} \epsilon_{i_{1} i_{4}} \epsilon_{i_{2} i_{3}}-\epsilon_{i_{1} i_{3}} \epsilon_{i_{2} i_{4}}\right), \\
\mathcal{O}_{4 f}^{4}= & \frac{1}{4 \Lambda_{\mathrm{TC}}^{2}}\left(\psi^{i_{1}}{ }_{a_{1}} \psi^{i_{2}}{ }_{a_{2}}\right)\left(\bar{\psi}^{i_{3} a_{3}} \bar{\psi}^{i_{4} a_{4}}\right) \\
& \times\left(\delta^{a_{1}}{ }_{a_{3}} \delta^{a_{2}}{ }_{a_{4}} \epsilon_{i_{1} i_{3}} \epsilon_{i_{2} i_{4}}+\delta^{a_{1}}{ }_{a_{4}} \delta^{a_{2}}{ }_{a_{3}} \epsilon_{i_{1} i_{4}} \epsilon_{i_{2} i_{3}}\right) \\
\mathcal{O}_{4 f}^{5}= & \frac{1}{4 \Lambda_{\mathrm{TC}}^{2}}\left(\psi^{i_{1}}{ }_{a_{1}} \psi^{i_{2}}{ }_{a_{2}}\right)\left(\bar{\psi}^{i_{3} a_{3}} \bar{\psi}^{i_{4} a_{4}}\right) \\
& \times\left(\delta^{a_{1}}{ }_{a_{3}} \delta^{a_{2}}{ }_{a_{4}} \epsilon_{i_{1} i_{4}} \epsilon_{i_{2} i_{3}}+\delta^{a_{1}}{ }_{a_{4}} \delta^{a_{2}}{ }_{a_{3}} \epsilon_{i_{1} i_{3}} \epsilon_{i_{2} i_{4}}\right)
\end{aligned}
$$

where $\bar{\psi}^{a i}=\epsilon^{i j} \bar{\psi}_{j}^{a}$. Note also that the above operators are self-conjugate. Similarly, one can construct five corresponding operators containing four left-handed spinors. However, we find that only three of them are truly independent, as shown in Appendix B. We take these three to be

$$
\begin{aligned}
& \mathcal{O}_{4 f}^{6}=\frac{1}{8 \Lambda_{\mathrm{TC}}^{2}}\left(\psi^{i_{1}}{ }_{a_{1}} \psi^{i_{2}}{ }_{a_{2}}\right)\left(\psi^{i_{3}}{ }_{3} \psi^{i_{4}}{ }_{a_{4}}\right) \\
& \times \Sigma^{a_{1} a_{2}} \sum^{a_{3} a_{4}} \epsilon_{i_{1} i_{2}} \epsilon_{i_{3} i_{4}}, \\
& \mathcal{O}_{4 f}^{7}=\frac{1}{8 \Lambda_{\mathrm{TC}}^{2}}\left(\psi^{i_{1}}{ }_{a_{1}} \psi^{i_{2}}{ }_{a_{2}}\right)\left(\psi^{i_{3}}{ }_{a_{3}} \psi^{i_{4}}{ }_{a_{4}}\right) \\
& \times\left(\Sigma^{a_{1} a_{4}} \Sigma^{a_{2} a_{3}}-\Sigma^{a_{1} a_{3}} \Sigma^{a_{2} a_{4}}\right) \epsilon_{i_{1} i_{2}} \epsilon_{i_{3} i_{4}}, \\
& \mathcal{O}_{4 f}^{8}=\frac{1}{8 \Lambda_{\mathrm{TC}}^{2}}\left(\psi^{i_{1}}{ }_{a_{1}} \psi^{i_{2}}{ }_{a_{2}}\right)\left(\psi^{i_{3}} a_{3} \psi_{a_{4}}^{i_{4}}\right) \\
& \times \Sigma^{a_{1} a_{2}} \sum^{a_{3} a_{4}}\left(\epsilon_{i_{1} i_{4}} \epsilon_{i_{2} i_{3}}-\epsilon_{i_{1} i_{3}} \epsilon_{i_{2} i_{4}}\right) .
\end{aligned}
$$

\footnotetext{
${ }^{3}$ The naming of these operators are loosely inspired by the corresponding operators in the SM effective field theory [29].
}
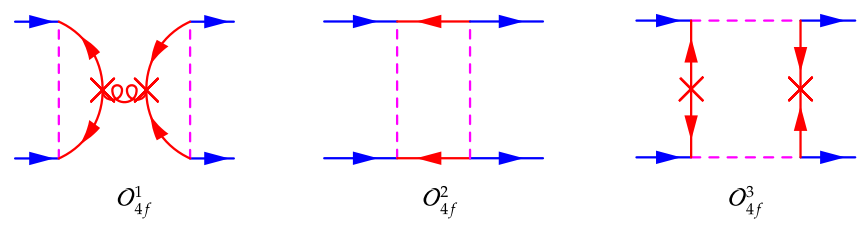

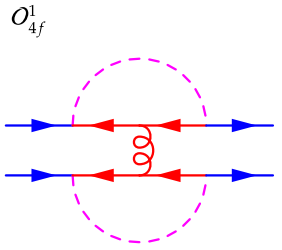

$O_{4 f}^{4}$

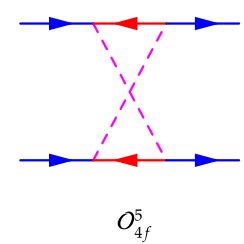

$O_{4 f}^{3}$

FIG. 1. Representative Feynman diagrams corresponding to the operators $\mathcal{O}_{4 f}^{1}-\mathcal{O}_{4 f}^{5}$ in Eqs. [(22)-(26)]. The blue colored lines are SM fermions, the red colored solid lines are TC fermions, the red colored curly lines are TC gluons, and the magenta lines are TC scalars.

For completeness, we also show the two-dependent operators

$$
\begin{aligned}
\mathcal{O}_{4 f}^{9}= & \frac{1}{8 \Lambda_{\mathrm{TC}}^{2}}\left(\psi^{i_{1}} a_{1} \psi_{a_{2}}^{i_{2}}\right)\left(\psi^{i_{3}} a_{3} \psi^{i_{4}} a_{4}\right) \\
& \times\left(\Sigma^{a_{1} a_{3}} \Sigma^{a_{2} a_{4}} \epsilon_{i_{1} i_{3}} \epsilon_{i_{2} i_{4}}+\Sigma^{a_{1} a_{4}} \Sigma^{a_{2} a_{3}} \epsilon_{i_{1} i_{4}} \epsilon_{i_{2} i_{3}}\right), \\
\mathcal{O}_{4 f}^{10}= & \frac{1}{8 \Lambda_{\mathrm{TC}}^{2}}\left(\psi^{i_{1}} a_{1} \psi^{i_{2}} a_{2}\right)\left(\psi^{i_{3}} a_{3} \psi^{i_{4}} a_{4}\right) \\
& \times\left(\Sigma^{a_{1} a_{3}} \sum^{a_{2} a_{4}} \epsilon_{i_{1} i_{4}} \epsilon_{i_{2} i_{3}}+\Sigma^{a_{1} a_{4}} \Sigma^{a_{2} a_{3}} \epsilon_{i_{1} i_{3}} \epsilon_{i_{2} i_{4}}\right),
\end{aligned}
$$

which are related to $\mathcal{O}_{4 f}^{6-8}$ via

$$
\mathcal{O}_{4 f}^{6}+\mathcal{O}_{4 f}^{9}=0, \quad \mathcal{O}_{4 f}^{7}+\mathcal{O}_{4 f}^{8}-\mathcal{O}_{4 f}^{10}=0 .
$$

For the case of $N_{\mathcal{F}}=4$ one can write another operator,

$\mathcal{O}_{A}=-\frac{1}{8 \Lambda_{\mathrm{TC}}^{2}}\left(\psi^{i_{1}} a_{1} \psi^{i_{2}} a_{2}\right)\left(\psi^{i_{3}} a_{3} \psi^{i_{4}} a_{4}\right) \epsilon^{a_{1} a_{2} a_{3} a_{4}} \epsilon_{i_{1} i_{2}} \epsilon_{i_{3} i_{4}}$, for

$N_{\mathcal{F}}=4$,

where $\epsilon^{a_{1} a_{2} a_{3} a_{4}}$ is the fully antisymmetric 4-index matrix which is naturally linked to the Adler-Bell-Jackiw anomaly of the global $\mathrm{U}(1)_{\mathcal{F}}$. However this operator is already contained in the list above because of the following operator identity:

$\mathcal{O}_{A}=\mathcal{O}_{4 f}^{10}-\mathcal{O}_{4 f}^{8}-\mathcal{O}_{4 f}^{9}=\mathcal{O}_{4 f}^{6}+\mathcal{O}_{4 f}^{7}$, for $N_{\mathcal{F}}=4$.

It is useful to represent each of the ten operators $\mathcal{O}_{4 f}^{1 \ldots 10}$ in terms of representative diagrams involving $\mathcal{F}$ and $\mathcal{S}$ loops, as shown in Figs. 1 and 2. Here the " $X$ " signifies an insertion of the dynamical TC-fermion mass, that is proportional to $\Sigma$. Thus the diagrams show how the $\Sigma$-dependence occurs in each operator. At a naive 

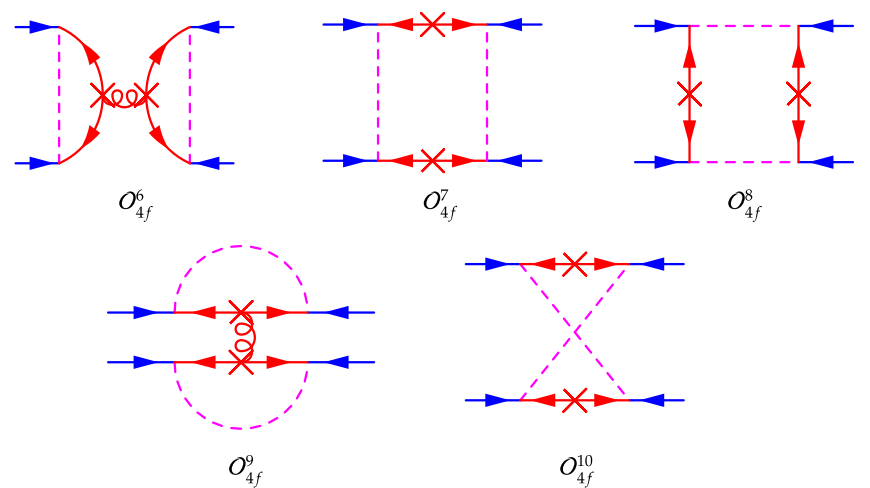

FIG. 2. Representative Feynman diagrams corresponding to the operators $\mathcal{O}_{4 f}^{6}-\mathcal{O}_{4 f}^{10}$ in Eqs. [(27)-(31)]. The blue colored lines are $\mathrm{SM}$ fermions, the red colored solid lines are TC fermions, the red colored curly lines are TC gluons, and the magenta lines are TC scalars.

perturbative level (these diagrams are only mnemonics) the operators $\mathcal{O}_{4 f}^{6-10}$ need mass insertion, while nonperturbatively one obtains operators such as $\mathcal{O}_{A}$ stemming from instanton corrections.

The case in which the masses of the scalars are much heavier than $\Lambda_{\mathrm{TC}}$ is obtained by replacing

$$
\epsilon_{i j} \rightarrow \Lambda_{\mathrm{TC}}^{2}\left(\frac{1}{M_{\mathcal{S}}^{2}}\right)_{i j}
$$

in each operator. The large mass limit corresponds physically to integrating out the scalars, which in the naive diagrams corresponds to replacing each heavy scalar propagator with the inverse mass matrix. Of course one needs to identify diagrammatically the leading contributions in the inverse scalar mass expansion, as shown in Figs. 1 and 2.

\section{Standard model loop-generated pNGB operators}

Loops of the elementary fermions are crucial in generating a potential for the pNGBs that includes the Higgs boson. As in other pNGB Higgs models, the potential contains radiative corrections that violate the global symmetries of the model once the spurionic Yukawa couplings assume their constant value. Accordingly, they play an important role in determining the vacuum alignment of the models. The simplest way to write down the fermion loop generated operators is to separate the Yukawa couplings $y_{f}$ from the elementary fermions: the Yukawa spurions thus inherit the same quantum numbers as $\psi$ under the global symmetries of the strong dynamics, but they also acquire transformation properties under the SM gauge symmetries as carried by the elementary fermions. If a SM fermion is in the representation $R_{\mathrm{SM}}$ of the SM gauge group then the corresponding $y_{f}$ transforms as

$$
\left(y_{f}\right)^{i}{ }_{a} \in \square_{\mathcal{S}} \otimes \bar{\square}_{\mathcal{F}} \otimes \bar{R}_{\mathrm{SM}},
$$

where, for simplicity, we do not explicitly write the gauge SM indices.

\section{Radiatively generated $p N G B$ potential}

At leading order in the chiral expansion, and quadratic order in the spurions, two operators might appear

$$
\begin{aligned}
& \frac{f_{\mathrm{TC}} \Lambda_{\mathrm{TC}}^{3}}{16 \pi^{2}}\left(y_{f}\right)^{i_{1}}{ }_{a_{1}}\left(y_{f^{\prime}}\right)^{i_{2}}{ }_{a_{2}} \Sigma^{a_{1} a_{2}} \epsilon_{i_{1} i_{2}}, \\
& \frac{f_{\mathrm{TC}} \Lambda_{\mathrm{TC}}^{3}}{16 \pi^{2}}\left(y_{f}^{*}\right)^{i_{1}, a_{1}}\left(y_{f^{\prime}}\right)^{i_{2}}{ }_{a_{2}} \delta_{a_{1}}{ }^{a_{2}} \epsilon_{i_{1} i_{2}} .
\end{aligned}
$$

However the latter is independent on the pNGB fields and therefore just an irrelevant constant in the potential, while the former is not SM gauge invariant and therefore is not generated. ${ }^{4}$

In contrast to the lack of operators at quadratic order in the spurions, there is a plethora of operators at quartic order. They involve loops of two SM fermions, each contracting the SM indices of two spurions $y_{f}$. Thus they all share the spurion structure

$$
\left(y_{f}^{*} y_{f}\right)^{a_{1}} a_{2} i_{1} i_{2},
$$

where the SM indices are contracted inside the parentheses and a sum over the SM fermions $f$ is left understood. This gives rise to three operators contributing to the pNGB potential,

$\mathcal{O}_{V_{f}}^{1}=\frac{f_{\mathrm{TC}}^{2} \Lambda_{\mathrm{TC}}^{2}}{16 \pi^{2}}\left(y_{f}^{*} y_{f}\right)^{a_{1}}{ }_{a_{2}}^{i_{1} i_{2}}\left(y_{f^{\prime}}^{*} y_{f^{\prime}}\right)^{a_{3}}{ }_{a_{4}}{ }^{i_{3} i_{4}} \sum_{a_{1} a_{3}}^{\dagger} \Sigma^{a_{2} a_{4}} \epsilon_{i_{1} i_{2}} \epsilon_{i_{3} i_{4}}$,

$\mathcal{O}_{V_{f}}^{2}=\frac{f_{\mathrm{TC}}^{2} \Lambda_{\mathrm{TC}}^{2}}{16 \pi^{2}}\left(y_{f}^{*} y_{f}\right)^{a_{1}}{ }_{a_{2}}{ }^{i_{1} i_{2}}\left(y_{f^{\prime}}^{*} y_{f^{\prime}}\right)^{a_{3}}{ }_{a_{4}}{ }_{i} i_{4} \Sigma_{4} \Sigma_{a_{1} a_{3}}^{\dagger} \Sigma^{a_{2} a_{4}} \epsilon_{i_{1} i_{3}} \epsilon_{i_{2} i_{4}}$,

$\mathcal{O}_{V_{f}}^{3}=\frac{f_{\mathrm{TC}}^{2} \Lambda_{\mathrm{TC}}^{2}}{16 \pi^{2}}\left(y_{f}^{*} y_{f}\right)^{a_{1}}{ }_{a_{2}}{ }^{i_{1} i_{2}}\left(y_{f^{\prime}}^{*} y_{f^{\prime}}\right)^{a_{3}}{ }_{a_{4}}{ }_{i_{3} i_{4}} \Sigma_{a_{1} a_{3}}^{\dagger} \Sigma^{a_{2} a_{4}} \epsilon_{i_{1} i_{4}} \epsilon_{i_{2} i_{3}}$,

all of which satisfy all symmetries. Again one may construct mnemonic, representative diagrams for the operators cf. Fig. 3. The factor of $16 \pi^{2}$ is a naive effort to account for the loops of the elementary fermions. One can think of these operators as coming from the three different ways of contracting the external SM fermions in operators $\mathcal{O}_{4 f}^{1,3}$.

\footnotetext{
${ }^{4}$ The former is due to the fact that the combination of Yukawas has the quantum numbers of mass terms for the SM fermions. Thus, the only term that may survive is proportional to the Majorana mass of right-handed neutrinos.
} 


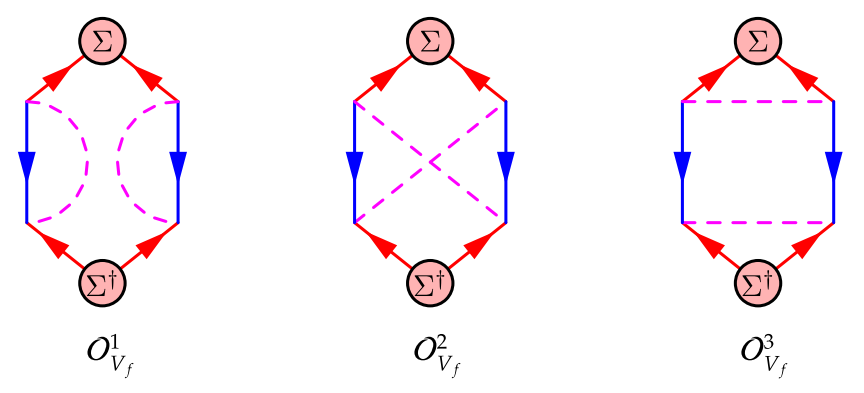

FIG. 3. Representative Feynman diagrams corresponding to the operators $\mathcal{O}_{V_{f}}^{1}-\mathcal{O}_{V_{f}}^{3}$ in Eqs. [(39)-(41)]. The blue colored lines are SM fermions, the red colored solid lines are TC fermions, and the magenta lines are TC scalars.

As in any other composite Higgs model there are contributions to the pNGB potential stemming from SM gauge bosons. At lowest order this is due to the operator,

$$
\mathcal{O}_{V_{g}}=\frac{f_{\mathrm{TC}}^{2} \Lambda_{\mathrm{TC}}^{2}}{16 \pi^{2}} \operatorname{Tr}\left[T_{\mathcal{F}}^{I} \Sigma\left(T_{\mathcal{F}}^{I}\right)^{\mathrm{T}} \Sigma^{\dagger}\right]
$$

Together with the $\chi_{+}$term in Eq. (15) stemming from the fundamental fermion masses, the operators mentioned in this section are responsible for the pNGB potential at leading order.

\section{Radiative corrections to the kinetic terms}

At NLO in the chiral expansion one finds corrections to the pNGB kinetic terms. We find a total of 21 such operators involving four $y_{f}$ spurions the full list of which can be found in Appendix C. Physically, they give corrections to the masses of the EW gauge bosons; however, we find that only six of them contribute to the oblique $T$ parameter. ${ }^{5}$ They are

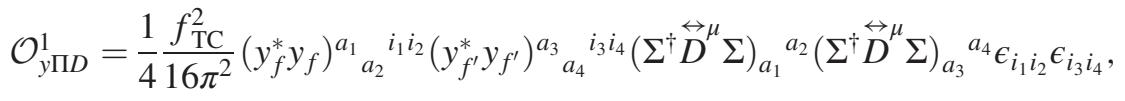

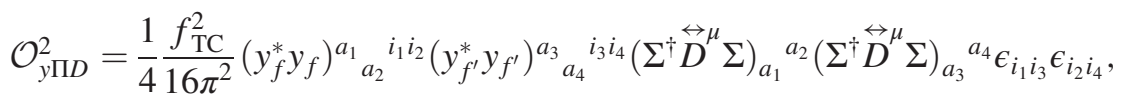

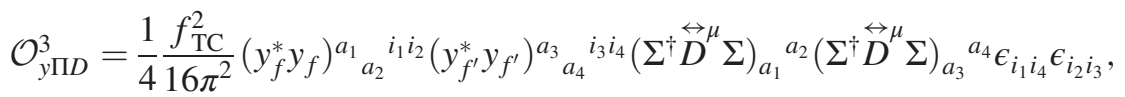

$$
\begin{aligned}
& \mathcal{O}_{y \Pi D}^{4}=\frac{1}{4} \frac{f_{\mathrm{TC}}^{2}}{16 \pi^{2}}\left(y_{f}^{*} y_{f}\right)^{a_{1}} a_{2}{ }_{a_{1} i_{2}}\left(y_{f^{\prime}}^{*} y_{f^{\prime}}\right)^{a_{3}} a_{4} i_{3} i_{4}\left(\Sigma^{\dagger} \stackrel{\leftrightarrow}{D}{ }^{\mu} \Sigma\right)_{a_{1}}{ }^{a_{4}}\left(\Sigma^{\dagger} \stackrel{\leftrightarrow}{D} \underline{\mu} \Sigma\right)_{a_{3}}{ }^{a_{2}} \epsilon_{i_{1} i_{2}} \epsilon_{i_{3} i_{4}}, \\
& \left.\mathcal{O}_{y \Pi D}^{5}=\frac{1}{4} \frac{f_{\mathrm{TC}}^{2}}{16 \pi^{2}}\left(y_{f}^{*} y_{f}\right)^{a_{1}} a_{2}{ }_{i_{1} i_{2}}\left(y_{f^{\prime}}^{*} y_{f^{\prime}}\right)^{a_{3}} a_{4} i_{3} i_{4}\left(\Sigma^{\dagger} \stackrel{\leftrightarrow}{D}{ }^{\mu} \Sigma\right)_{a_{1}}{ }^{a_{4}}\left(\Sigma^{\dagger} \stackrel{\leftrightarrow}{D} \Sigma\right)\right)_{a_{3}}{ }^{a_{2}} \epsilon_{i_{1} i_{3}} \epsilon_{i_{2} i_{4}}, \\
& \mathcal{O}_{y \Pi D}^{6}=\frac{1}{4} \frac{f_{\mathrm{TC}}^{2}}{16 \pi^{2}}\left(y_{f}^{*} y_{f}\right)^{a_{1}} a_{2}{ }^{i_{1} i_{2}}\left(y_{f^{\prime}}^{*} y_{f^{\prime}}\right)^{a_{3}} a_{4}{ }^{i_{3} i_{4}}\left(\Sigma^{\dagger} \stackrel{\leftrightarrow}{D} \stackrel{\leftrightarrow}{\mu}^{\mu}\right)_{a_{1}}{ }^{a_{4}}\left(\Sigma^{\dagger} \stackrel{\leftrightarrow}{D}{ }^{\mu} \Sigma\right)_{a_{3}}{ }^{a_{2}} \epsilon_{i_{1} i_{4}} \epsilon_{i_{2} i_{3}} .
\end{aligned}
$$

These operators can be visualized as loops of TC-scalars and SM fermions, with TC-fermions in the external legs that close on meson fields and currents.

Again, for completeness, we note the SM gauge corrections to pNGB kinetic term. From one propagating gauge bosons, there are two operators which contribute to the $T$ parameter,

$$
\begin{aligned}
& \mathcal{O}_{\Pi D}^{1}=\frac{1}{4} \frac{f_{\mathrm{TC}}^{2}}{16 \pi^{2}} \operatorname{Tr}\left[\left(\Sigma \stackrel{\leftrightarrow}{D}_{\mu} \Sigma^{\dagger}\right) T_{\mathcal{F}}^{I}\left(\Sigma \stackrel{\leftrightarrow}{D}^{\mu} \Sigma^{\dagger}\right) T_{\mathcal{F}}^{I}\right], \\
& \mathcal{O}_{\Pi D}^{2}=\frac{1}{4} \frac{f_{\mathrm{TC}}^{2}}{16 \pi^{2}} \operatorname{Tr}\left[\left(\stackrel{\leftrightarrow}{D}_{\mu} \Sigma^{\dagger}\right) T_{\mathcal{F}}^{I}\right] \operatorname{Tr}\left[\left(\Sigma \stackrel{\leftrightarrow}{D}^{\mu} \Sigma^{\dagger}\right) T_{\mathcal{F}}^{I}\right]
\end{aligned}
$$

\footnotetext{
${ }^{5}$ Assuming couplings to all SM fermions and right-handed neutrinos with fundamental Yukawa couplings as given in Eq. (68).
}

Here there is an implicit sum over all the gauge bosons $I$, and a trace over the $\mathrm{SU}\left(N_{\mathcal{F}}\right)$ index. The full list can again be found in Appendix C.

Furthermore, also at NLO in the chiral expansion the operator

$$
\mathcal{O}_{W W}=\frac{f_{\mathrm{TC}}^{2}}{2 \Lambda_{\mathrm{TC}}^{2}} A_{\mu \nu}^{I} A^{J \mu \nu} \operatorname{Tr}\left[T_{\mathcal{F}}^{I} \Sigma\left(T_{\mathcal{F}}^{J}\right)^{\mathrm{T}} \Sigma^{\dagger}\right]
$$

gives the only contribution to the $S$ parameter.

\section{TOP AND BOTTOM PHYSICS IN THE MOST MINIMAL MODEL OF FUNDAMENTAL PARTIAL COMPOSITENESS}

We now specialize to the most minimal model [11], defined by the choice of gauge group $G_{\mathrm{TC}}=\mathrm{SU}(2) \sim$ $\operatorname{Sp}(2)$ and $N_{\mathcal{F}}=4$ Weyl TC-fermions in the fundamental 
TABLE II. Fundamental technicolor states with their gauge quantum numbers and global symmetries. The table includes the third generation quarks too and the charge assignment under the baryon number $\mathrm{U}(1)_{B}$.

\begin{tabular}{lcccccc}
\hline \hline & $\mathrm{SU}(3)_{c}$ & $\mathrm{SU}(2)_{\mathrm{L}}$ & $\mathrm{U}(1)_{Y}$ & $\mathrm{U}(1)_{B}$ & $\mathrm{SU}(4)_{\mathcal{F}}$ & $\mathrm{Sp}(6)_{\mathcal{S}}$ \\
\hline $\mathcal{F}_{Q}$ & 1 & $\square$ & 0 & & & \\
$\mathcal{F}_{u}$ & 1 & 1 & $-\frac{1}{2}$ & 0 & $\square$ & 1 \\
$\mathcal{F}_{d}$ & 1 & 1 & $\frac{1}{2}$ & & & \\
$\mathcal{S}_{t}$ & $\square$ & 1 & $-\frac{1}{6}$ & $-\frac{1}{3}$ & 1 & $\square$ \\
$Q_{3}$ & $\square$ & $\square$ & $\frac{1}{6}$ & $\frac{1}{3}$ & & \\
$u_{3}$ & $\square$ & 1 & $-\frac{2}{3}$ & $-\frac{1}{3}$ & & \\
$d_{3}$ & $\square$ & 1 & $\frac{1}{3}$ & $-\frac{1}{3}$ & & \\
\hline \hline
\end{tabular}

representation. We start the analysis by studying in detail the minimal TC-scalar sector to give mass to top and bottom alone. The TC-scalar sector, therefore, only contains a single field $\mathcal{S}_{t}$, with quantum numbers summarized in Table II: the global symmetry is $\operatorname{Sp}(6)$ since $N_{\mathcal{S}}=3$. With respect to the SM gauge group $G_{\mathrm{SM}}$, the Weyl TCfermions transform as $\mathcal{F}_{Q} \in(1,2)_{0}, \mathcal{F}_{u} \in(1,1)_{-1 / 2}$, and $\mathcal{F}_{d} \in(1,1)_{1 / 2}$. The overall theory is gauge anomaly free. Note that the fermionic sector of this TC model was originally proposed in Refs. [30,31]. The vacuum alignment of the theory can be written as the following antisymmetric matrix in the $\mathrm{SU}(4)$ space [19]:

$$
\Sigma_{0}^{a b}=\left(\begin{array}{cccc}
0 & c_{\theta} & s_{\theta} & 0 \\
-c_{\theta} & 0 & 0 & s_{\theta} \\
-s_{\theta} & 0 & 0 & -c_{\theta} \\
0 & -s_{\theta} & c_{\theta} & 0
\end{array}\right)
$$

The angle $\theta$ parametrizes the alignment of the vacuum with respect to the EW embedding [32] and relates the pNGB decay constant to the EW scale as $v_{\mathrm{EW}}=f_{\mathrm{TC}} s_{\theta}=f_{\mathrm{TC}} \sin \theta$.

At the fundamental Lagrangian level the new Yukawa couplings with the SM fields read

$$
\begin{aligned}
\mathcal{L}_{\text {top-bottom }}= & y_{Q_{3}} Q_{3, \alpha} \mathcal{S}_{t} \epsilon_{\mathrm{TC}} \mathcal{F}_{Q}^{\alpha}-y_{t} u_{3} \mathcal{S}_{t}^{*} \mathcal{F}_{d} \\
& +y_{b} d_{3} \mathcal{S}_{t}^{*} \mathcal{F}_{u}+\text { H.c. },
\end{aligned}
$$

where $\alpha$ is the $\mathrm{SU}(2)_{\mathrm{L}}$ index, and $u_{3}$ and $d_{3}$ are the lefthanded spinors constructed out of the charge-conjugate right-handed top and bottom singlets. The above Yukawa interactions can be written in the compact form of Eq. (6) by defining a spurion

$$
\psi^{i}{ }_{a}=\left(\begin{array}{cccc}
0 & 0 & y_{b} d_{3} & -y_{t} u_{3} \\
y_{Q_{3}} q_{3}^{(d)} & -y_{Q_{3}} q_{3}^{(u)} & 0 & 0
\end{array}\right),
$$

where each row transforms as antifundamental of $\mathrm{SU}(4)_{\mathcal{F}}$ and each column as a fundamental of $\operatorname{Sp}(6)_{\mathcal{S}}{ }^{6}$. Note that $Q_{3, \alpha}=\varepsilon_{\alpha \beta} Q_{3}^{\beta}=\left(-q_{3}^{(d)}, q_{3}^{(u)}\right)$ transforms as an antidoublet of $\mathrm{SU}(2)_{\mathrm{L}}$, while $\left(y_{b} d_{3},-y_{t} u_{3}\right)$ as a doublet of $\mathrm{SU}(2)_{\mathrm{R}}$, consistently with the decomposition of an $\square$ of $\mathrm{SU}(4)_{\mathcal{F}}$.

The operator $\mathcal{O}_{\text {Yuk }}$, in Eq. (18), is responsible for the generation of the SM fermion masses and Yukawa couplings to the Higgs boson (up to the effects of nonlinearities in the pNGB fields),

$$
\begin{aligned}
\mathcal{L}_{\mathrm{EFT}} \supset & -C_{\mathrm{Yuk}} v_{\mathrm{EW}}\left(y_{Q_{3}} y_{b} q_{3}^{(d)} d_{3}+y_{Q_{3}} y_{t} q_{3}^{(u)} u_{3}\right) \\
& \times\left(1+\frac{c_{\theta} h}{v_{\mathrm{EW}}}+\cdots\right)+\text { H.c. }
\end{aligned}
$$

The top and bottom masses can, thus, be identified with

$m_{t}=\left|C_{\mathrm{Yuk}} y_{Q_{3}} y_{t}\right| v_{\mathrm{EW}}$ and $m_{b}=\left|C_{\mathrm{Yuk}} y_{Q_{3}} y_{b}\right| v_{\mathrm{EW}}$.

A potential for the Higgs boson, and the other pNGB, generated by loops of top and bottom, is encoded in the operators in Eqs. (39)-(41). Expanding in the pNGB fields, the term that corresponds to a potential for the alignment angle $\theta$ reads

$$
\begin{aligned}
V_{\mathrm{t} / \mathrm{b}}(\theta)= & -\frac{3 f_{\mathrm{TC}}^{2} \Lambda_{\mathrm{TC}}^{2}}{8 \pi^{2}}\left[\left|y_{Q_{3}}\right|^{2}\left(\left|y_{t}\right|^{2}+\left|y_{b}\right|^{2}\right)\left(3 C_{V_{f}}^{1}+C_{V_{f}}^{2}\right) s_{\theta}^{2}\right. \\
& \left.+\left(\left|y_{Q_{3}}\right|^{4}+\left|y_{t} y_{b}\right|^{2}\right)\left(3 C_{V_{f}}^{1}-C_{V_{f}}^{3}\right) c_{\theta}^{2}\right] .
\end{aligned}
$$

This first term, proportional to $s_{\theta}^{2}$, has the same form as the contribution generated by a direct bilinear coupling of the top and bottom to the TC-fermions, as used in Ref. [19,31]: the combinations of Yukawas are, in fact, proportional to the top and bottom masses. As usual, expecting a negative sign in front coming from the fermion loop, this term alone tends to destabilize the vacuum alignment towards the TC $\operatorname{limit} \theta=\pi / 2$. The second term, proportional to $c_{\theta}^{2}$, is new in FPC models and, depending on the sign of the coefficients, it may either contribute to the destabilization or tend to flip the alignment to the EW preserving direction. To achieve electroweak symmetry breaking one should have $\left|y_{Q_{3}}\right|<\left|y_{t}\right|$, which, as we shall see, is supported by the constraints coming from the $Z$ boson to $\bar{b} b$.

The potential also receives contributions from the gauge interactions, encoded in Eq. (42), and the TC-fermion mass, as shown in Eq. (15), which have the same form as in models without FPC $[19,31,33]$. In particular, the contribution of the TC-fermion mass can be used to stabilize the potential around small $\theta$ values against the top loops, in order to obtain a pNGB Higgs boson. Note that higher dimension operators generated by top loops may also help

\footnotetext{
${ }^{6}$ The implicit QCD color indices of the quarks are embedded as part of $\operatorname{Sp}(6)$.
} 
stabilizing the potential; however they are expected to be subleading.

\section{A. Couplings of the $Z$ to the bottom quark}

We now turn to the operator in Eq. (19) that generates corrections to the gauge couplings of the massive gauge bosons to fermions,

$$
\begin{aligned}
\mathcal{O}_{\Pi f}= & \frac{g}{2 \cos \theta_{W}} \frac{f_{\mathrm{TC}}}{\Lambda_{\mathrm{TC}}} s_{\theta}^{2} Z_{\mu}\left(\left|y_{Q_{3}}\right|^{2}\left(\bar{t}_{\mathrm{L}} \gamma^{\mu} t_{\mathrm{L}}-\bar{b}_{\mathrm{L}} \gamma^{\mu} b_{\mathrm{L}}\right)\right. \\
& \left.\quad+\left|y_{b}\right|^{2} \bar{b}_{\mathrm{R}} \gamma^{\mu} b_{\mathrm{R}}-\left|y_{t}\right|^{2} \bar{t}_{\mathrm{R}} \gamma^{\mu} t_{\mathrm{R}}\right) \\
& -\frac{g}{\sqrt{2}} \frac{f_{\mathrm{TC}}}{\Lambda_{\mathrm{TC}}} s_{\theta}^{2} W_{\mu}^{+}\left(y_{b}^{*} y_{t} \bar{t}_{\mathrm{R}} \gamma^{\mu} b_{\mathrm{R}}-\left|y_{Q_{3}}\right|^{2} \bar{t}_{\mathrm{L}} \gamma^{\mu} b_{\mathrm{L}}\right)+\text { H.c. }
\end{aligned}
$$

where the SM top and bottom are in the usual Dirac spinor notation. While the couplings of the top to the $Z$ are unconstrained, and $y_{b}$ can be taken small to reproduce the bottom mass, the coupling of the left-handed bottom to the $Z$ receives sizeable corrections proportional to $\left|y_{Q_{3}}\right|^{2}$. The well-known issue is that $y_{Q_{3}}$ coupling cannot be too small, as it enters the formula for the top mass. Imposing the latest constraints $[34,35]$, we obtain the $2 \sigma$ limit, $^{7,8}$

$$
C_{\Pi f}\left|y_{Q_{3}}\right|^{2} s_{\theta}^{2}<0.043, \quad @ 95 \% \text { C.L. }
$$

This constraint mainly comes from the measurement of $R_{b}$ at LEP [36]. The constraint on $\theta$ from electroweak precision tests tends to ease the tension, as $s_{\theta}^{2} \lesssim 0.1$ is generically required [37]. Furthermore, it is possible to obtain the correct top mass with a small $y_{Q_{3}}$ by maximizing the right-handed mixing $y_{t}$; i.e., assuming that the right-handed top is more composite than the left-handed part. Interestingly, this configuration is also preferred in the top-loop induced potential for the alignment of the vacuum, as we have seen in (57). Using Eq. (56), the above bound translates into the following lower bound on the right-handed top mixing 9 :

$$
\left|y_{t}\right| \frac{\left|C_{\mathrm{Yuk}}\right|}{\sqrt{C_{\Pi f}}} \gtrsim \frac{m_{t}}{f_{\mathrm{TC}}} \frac{1}{\sqrt{0.043}}=\frac{10 \mathrm{TeV}}{\Lambda_{\mathrm{TC}}},
$$

which, for reasonably low scale compositeness, $\Lambda_{\mathrm{TC}}=$ $10 \mathrm{TeV}$, and $C_{\mathrm{Yuk}}=C_{\Pi f}=1$, corresponds to the bound $\left|y_{t}\right| \gtrsim 1$. This implies that the fundamental Yukawa coupling would have to be larger than $2 \sqrt{\pi} \sim 3.5$, as discussed in Sec. II. It should be mentioned that it is enough that one (or both) strong coefficient departs from unity by a factor of a

\footnotetext{
${ }^{7}$ For all our numerical estimates we have used $\Lambda_{\mathrm{TC}}=4 \pi f_{\mathrm{TC}}$.

${ }^{8}$ Please note that all bounds found here, are on the effective rather than the fundamental Yukawa parameters.

${ }^{9}$ Note that our normalization for the pre-Yukawa couplings differs from the one usually considered in EFT realizations, see Sec. IV A for more details.
}

few to lower the bound on the fundamental Yukawa coupling, thus allowing for perturbative values at the condensation scale.

A possible concern for the model is that the fundamental Yukawa couplings may become large enough that they cause an unwanted condensate, $\langle f \mathcal{F}\rangle$, to form between technifermions and SM fermions [breaking both SU(3) color and TC]. An approximate Schwinger-Dyson analysis for a Yukawa model with $\mathrm{SU}(2)_{\mathrm{L}} \times \mathrm{SU}(2)_{\mathrm{R}}$ symmetry would indicate that such a condensate only forms for $y \gtrsim O(2 \pi)$ $[38,39]$. If this estimate is also valid in the MFPC, it would suggest that the model may be safe from forming a Yukawa induced condensate. However, further work is required to verify this, e.g., on the lattice. Once more, suitable $O$ (few) strong coefficients would render this potential problem mute.

\section{B. Effective interactions for the top sector}

The effective Lagrangian for EW physics contains four fermion interactions which are induced by the underlying strong dynamics. In Sec. II C, we showed that there are eight independent operators, five of which are self-Hermitian. Expanding the operators $\mathcal{O}_{4 f}^{i}$ we obtain four-fermion interactions involving the SM fermions listed in Appendix D. Note that these set of operators cannot be directly matched to the Warsaw basis [29] because our theory contains nonlinearities in the Higgs field. Effectively, this gives us the Wilson coefficient for each operator in terms of the fundamental Yukawa couplings, the scale of strong dynamics $\Lambda_{\mathrm{TC}}$, and the coefficients $C_{4 f}^{i}$ of the strong dynamics.

The phenomenologically relevant operators involve four tops, as they are directly probed at the LHC in four top final states, such as

$$
\begin{aligned}
\mathcal{L}_{\mathrm{EFT}} & \supset \frac{C_{4 f}^{4}+C_{4 f}^{5}}{4 \Lambda_{\mathrm{TC}}^{2}}\left|y_{t}\right|^{4}\left(\bar{t}_{\mathrm{R}} \gamma^{\mu} t_{\mathrm{R}}\right)\left(\bar{t}_{\mathrm{R}} \gamma_{\mu} t_{\mathrm{R}}\right) \\
& =\frac{C_{4 f}^{4}+C_{4 f}^{5}}{4 \Lambda_{\mathrm{TC}}^{2}}\left|y_{t}\right|^{4} O_{u u}^{3333},
\end{aligned}
$$

where the four 3's refer to the generation of each of the four fermions. ATLAS [40] puts an upper limit on this operator at $95 \%$ C.L., yielding the constraint,

$$
\begin{aligned}
& \frac{\left|C_{4 f}^{4}+C_{4 f}^{5}\right|}{4 \Lambda_{\mathrm{TC}}^{2}}\left|y_{t}\right|^{4}<2.9 \mathrm{TeV}^{-2} \\
& \Rightarrow\left|C_{4 f}^{4}+C_{4 f}^{5}\right|^{1 / 4}\left|y_{t}\right|<5.8\left(\frac{\Lambda_{\mathrm{TC}}}{10 \mathrm{TeV}}\right)^{1 / 2}, \quad @ 95 \% \text { C.L. }
\end{aligned}
$$

The above upper bound is compatible with the lower bound in Eq. (60), and the situation improves significantly for increasing values of $\Lambda_{\mathrm{TC}}$.

In addition to the four fermion interactions, the operators $\mathcal{O}_{f W}$ and $\mathcal{O}_{f G}$, in Eqs. (20) and (21), give rise to new dipole 
interactions between gauge fields and SM fermions. Knowing that the SM gauge bosons are embedded in the two global symmetries $\mathrm{SU}(4)_{\mathcal{F}}$ and $\mathrm{Sp}(6)_{\mathcal{S}}$ in the following way:

$$
\begin{aligned}
A_{\mu}^{I}\left(T_{\mathcal{F}}^{I}\right)^{a}{ }_{b} & =\frac{1}{2}\left(\begin{array}{cc}
g W_{\mu}^{i} \tau^{i} & 0 \\
0 & -g^{\prime} B_{\mu} \tau^{3}
\end{array}\right) \text { and } \\
G_{\mu}^{A}\left(T_{\mathcal{S}}^{A}\right)_{j}^{i} & =\frac{g_{S}}{2} G_{\mu}^{A}\left(\begin{array}{cc}
-\lambda^{* A} & 0 \\
0 & \lambda^{A}
\end{array}\right)+\frac{g^{\prime}}{6} B_{\mu}\left(\begin{array}{cc}
-\mathbb{1} & 0 \\
0 & \mathbb{1}
\end{array}\right),
\end{aligned}
$$

where $W, B$ and $G$ represent respectively the $\mathrm{SU}(2)_{\mathrm{L}}$, hypercharge and QCD gauge bosons respectively, the operators generate the following couplings:

$$
\begin{aligned}
& \mathcal{O}_{f W}=\frac{-1}{C_{\mathrm{Yuk}} \Lambda_{\mathrm{TC}}^{2}} \frac{m_{t}}{2 \sqrt{2} v_{\mathrm{EW}}}\left(g \mathcal{O}_{u W}^{33 *}+g^{\prime} \mathcal{O}_{u B}^{33 *}\right)+\cdots \\
& \mathcal{O}_{f G}=\frac{-1}{C_{\mathrm{Yuk}} \Lambda_{\mathrm{TC}}^{2}} \frac{\sqrt{2} m_{t}}{v_{\mathrm{EW}}}\left(g_{s} \mathcal{O}_{u G}^{33 *}+\frac{g^{\prime}}{6} \mathcal{O}_{u B}^{33 *}\right)+\cdots,
\end{aligned}
$$

where the Yukawa couplings have been expressed in terms of the physical top mass, as in Eq. (56). The dots contain couplings of the pNGBs generated by the nonlinearities, and the operators $\mathcal{O}_{u V}^{33}$ are from the SM EFT [41]. The TopFitter Collaboration [42] has extracted constraints on the anomalous couplings of the top quarks, in the EFT language, by considering the latest data on top production cross sections and distributions. The bound on $\mathcal{O}_{u B}^{33}$ is weaker than that on $\mathcal{O}_{u W}^{33}$, so we can use the latter to impose bounds on $C_{f W}$,

$$
\left|\frac{C_{f W}}{C_{\mathrm{Yuk}}}\right|<2500\left(\frac{\Lambda_{\mathrm{TC}}}{10 \mathrm{TeV}}\right)^{2} @ 95 \% \text { C.L. }
$$

The bound from the gluon coupling $\mathcal{O}_{u G}^{33}$ yields a stronger bound, ${ }^{10}$

$$
\left|\frac{C_{f G}}{C_{\mathrm{Yuk}}}\right|<110\left(\frac{\Lambda_{\mathrm{TC}}}{10 \mathrm{TeV}}\right)^{2} @ 95 \% \text { C.L. }
$$

Both of these constraints are obtained from marginalized bounds on the operators. Limiting other operators may therefore lead to stronger bounds.

\section{Extension to light generations and leptons}

The fundamental Lagrangian can be expanded to include all three generations of quarks and leptons. The minimal strategy [11] is to extend the TC-scalar sector by three extra

\footnotetext{
${ }^{10}$ The bounds come from the 95\% C.L. limits on the SM EFT operator coefficients $\frac{v_{\mathrm{EW}}^{2}}{\Lambda^{2}}\left|C_{u W}^{33}\right|<0.242$ and $\frac{v_{\mathrm{EW}}^{2}}{\Lambda^{2}}\left|C_{u G}^{33}\right|<0.079$ from Ref. [42].
}

TABLE III. Fundamental technicolor states and SM fermions with their SM gauge quantum numbers. The table also includes the charge assignments under the baryon and lepton number $\mathrm{U}(1)_{B, \ell}$.

\begin{tabular}{lccccccc}
\hline \hline & $\mathrm{SU}(3)_{c}$ & $\mathrm{SU}(2)_{\mathrm{L}}$ & $\mathrm{U}(1)_{Y}$ & $\mathrm{U}(1)_{B}$ & $\mathrm{U}(1)_{\ell}$ & $\mathrm{U}(3)_{g_{1}}$ & $\mathrm{U}(3)_{g_{2}}$ \\
\hline $\mathcal{F}_{Q}$ & 1 & $\square$ & 0 & & & & \\
$\mathcal{F}_{u}$ & 1 & 1 & $-\frac{1}{2}$ & 0 & 0 & 1 & 1 \\
$\mathcal{F}_{d}$ & 1 & 1 & $\frac{1}{2}$ & & & & \\
$\mathcal{S}_{q}$ & $\square$ & 1 & $-\frac{1}{6}$ & $-\frac{1}{3}$ & 0 & $\square$ & 1 \\
$\mathcal{S}_{l}$ & 1 & 1 & $\frac{1}{2}$ & 0 & -1 & 1 & $\square$ \\
$Q$ & $\square$ & $\square$ & $\frac{1}{6}$ & $\frac{1}{3}$ & 0 & & \\
$u$ & $\square$ & 1 & $-\frac{2}{3}$ & $-\frac{1}{3}$ & 0 & & \\
$d$ & $\square$ & 1 & $\frac{1}{3}$ & $-\frac{1}{3}$ & 0 & & \\
$L$ & 1 & $\square$ & $-\frac{1}{2}$ & 0 & 1 & & \\
$e$ & 1 & 1 & -1 & 0 & -1 & & \\
$\nu$ & 1 & 1 & 0 & 0 & -1 & & \\
\hline \hline
\end{tabular}

uncolored scalars $\mathcal{S}_{l}$ to couple to the three generations of leptons, and two extra colored scalars $\mathcal{S}_{u}$ and $\mathcal{S}_{c}$ (corresponding to six complex scalars) to couple to the two light quark generations. In total, therefore, we have $N_{\mathcal{S}}=12$ complex scalars, which enjoy a global $\mathrm{Sp}(24)_{\mathcal{S}}$ symmetry. The quantum numbers of both the TC and the SM fields are summarized in Table III.

The complete Yukawa interactions now read ${ }^{11}$

$$
\begin{aligned}
\mathcal{L}_{\text {yuk }}= & y_{Q} Q_{\alpha} \mathcal{S}_{q} \epsilon_{\mathrm{TC}} \mathcal{F}_{Q}^{\alpha}-y_{u} u \mathcal{S}_{q}^{*} \mathcal{F}_{d}+y_{d} d \mathcal{S}_{q}^{*} \mathcal{F}_{u} \\
& +y_{\ell} L_{\alpha} \mathcal{S}_{l} \epsilon_{\mathrm{TC}} \mathcal{F}_{Q}^{\alpha}-y_{\nu} \nu \mathcal{S}_{l}^{*} \mathcal{F}_{d}+y_{e} e \mathcal{S}_{l}^{*} \mathcal{F}_{u} \\
& -\tilde{y}_{\nu} \nu \mathcal{S}_{l} \epsilon_{\mathrm{TC}} \mathcal{F}_{u}+\text { H.c. },
\end{aligned}
$$

where each coupling is a $3 \times 3$ matrix in flavor space, and the flavor indices are left implicit for readability. Table III also contains the symmetries $U(3)_{g_{1,2}}$ corresponding to global approximate flavor symmetries between the three generations of each TC scalar. Additionally the full model still preserves a baryon number symmetry as does the SM. However, the lepton number symmetry is explicitly violated by the coupling $\tilde{y}_{\nu}$, not surprisingly as the inclusion of such a coupling gives rise to a Majorana mass term for the right-handed neutrinos.

Just as in the case of the top and bottom, the Yukawa interactions can be written in the more compact form from Eq. (6) by defining the spurion field $\psi$ as (color and generation indices are, once again, left implicit)

\footnotetext{
${ }^{11}$ Note that the scalars are in the conjugate representation of $G_{\mathrm{SM}}$ as compared to the minimal model suggested in Ref. [11].
} 


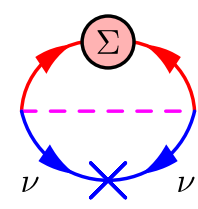

FIG. 4. Representative diagram for the contribution to the Higgs potential of a right-handed neutrino with an elementary Majorana mass, symbolized here by a blue cross.

$$
\psi_{a}^{i}=\left(\begin{array}{cccc}
0 & 0 & y_{d} d & -y_{u} u \\
0 & 0 & y_{e} e & -y_{\nu} \nu \\
y_{Q} q^{(d)} & -y_{Q} q^{(u)} & 0 & 0 \\
y_{\ell} l^{(e)} & -y_{\ell} l^{(\nu)} & \tilde{y}_{\nu} \nu & 0
\end{array}\right),
$$

where $a \in \mathrm{SU}(4)_{\mathcal{F}}$ and $i \in \mathrm{Sp}(24)_{\mathcal{S}}$. Details of this construction are found in Appendix A. The hierarchy of the fermion masses can be encoded either in the fundamental Yukawa couplings or in a hierarchy in the mass spectrum of the TC-scalars. The phenomenology of the two scenarios is different for the low energy flavor observables as well as for the spectrum of the massive composite states of the theory. It is noteworthy that, thanks to the compact spurion form, the effect of the light generations can be expressed in terms of the same operator basis we used for the top/bottom case. Of course, at the EW scale the effect of light quarks will be negligible, as they are suppressed by the small effective Yukawas (or scalar masses), and we leave the effects on low energy flavor physics and lepton masses for further investigations.

The only exception is given by the physics of the righthanded neutrinos that might have Majorana masses and order-1 fundamental Yukawa couplings. Note that the presence of both Yukawas $y_{\nu}$ and $\tilde{y}_{\nu}$ will also generate a composite Majorana mass for the right-handed neutrino of the order $C_{\mathrm{Yuk}} c_{\theta} f_{\mathrm{TC}} y_{\nu} \tilde{y}_{\nu}$. At the same time, the first operator in Eq. (37) gives rise to a nonvanishing contribution to the Higgs potential

$$
V_{\nu}(\theta) \sim-\frac{f_{\mathrm{TC}} \Lambda_{\mathrm{TC}}^{3}}{8 \pi^{2}} y_{\nu} \tilde{y}_{\nu} c_{\theta},
$$

which only exists if an elementary Majorana mass is present. A mnemonic diagram for this operator is sketched in Fig. 4. This term has the same dependence on the alignment angle $\theta$ as the contribution of the TC fermion mass [19]; thus it can be used to stabilize the potential generated by the top loops towards small values of $\theta$ if the Yukawa couplings of at least one neutrino are of order 1 . This would provide a new mechanism where partial compositeness for neutrinos generates both $\mathrm{TeV}$-scale seesaw and stabilizes the Higgs potential.

\section{CONNECTIONS WITH OTHER APPROACHES TO PARTIAL COMPOSITENESS}

In this section we sketch the connection between our analysis, and other approaches used in the literature to study partial compositeness. We first address effective approaches, based either on the construction of an EFT or on extra dimensional implementations. Finally, we comment on the possible applicability of our results to purely fermionic underlying theories featuring partial compositeness.

\section{A. Effective operator approach}

The most popular approach to composite Higgs models in the literature has been to construct EFTs simply based on the symmetry breaking patterns (see Refs. [43,44] for a pedagogical introduction), without any reference to the underlying theory. ${ }^{12}$ As a consequence, to implement partial compositeness, the choice of the representation under which the top partners transform has been arbitrary. Furthermore, top partners in the EFT approach have been assumed to be the main driving force in the stabilization of the vacuum alignment along the small- $\theta$ limit: this mechanism can only work if the top partners are light $[45,46]$ and the contribution to the pNGB potential is dominated by their loops. Accepting the lightness of top partners with respect to the natural resonance scale, i.e., $\Lambda_{\mathrm{TC}} \sim 4 \pi f_{\mathrm{TC}}$, one is justified to include them in the EFT construction. Note however that top partners are not necessarily the only contributors to the Higgs potential $[19,31,33]$.

In the case under study in this work, the representation of the top partners is fixed to be the fundamental of the global symmetry SU(4). This choice has been considered problematic in the literature, as it typically leads to large corrections to the $Z$ coupling to bottoms. However, as we will see shortly, this problem only applies if the top partners are light. It is instructive to compare our general operator approach presented in Sec. III with the results one would obtain by adding the top partners to the EFT. The couplings of the top partners, that we collectively call $\mathcal{B}$, to the SM fermions can be written as

$$
\begin{aligned}
\mathcal{L}_{\mathrm{PC}} & =-\bar{y}_{Q_{3}}^{\mathrm{EFT}} f_{\mathrm{TC}} \bar{\psi}_{Q_{3}} \cdot \Sigma^{\dagger} \cdot \mathcal{B}_{R}-\bar{y}_{t}^{\mathrm{EFT}} f_{\mathrm{TC}} \overline{\mathcal{B}}_{L} \cdot \Sigma \cdot \psi_{t}, \\
\text { with } \psi_{Q_{3}} & =\left(\begin{array}{c}
Q_{3} \\
0 \\
0
\end{array}\right) \text { and } \psi_{t}=\left(\begin{array}{c}
0 \\
u_{3} \\
0
\end{array}\right),
\end{aligned}
$$

where the SM fermions are embedded into spurions transforming as the fundamental of $\mathrm{SU}(4)_{\mathcal{F}}$. The symmetries associated to the scalars $\mathcal{S}$ are thus ignored. The mass of the top can be obtained by diagonalizing the resulting mass matrix, yielding

\footnotetext{
${ }^{12}$ This approach might be the only available one if the underlying theory is conformal, in which case it can only be defined in terms of operators and their conformal dimensions.
} 


$$
m_{t}=2 M_{\mathcal{B}} s_{\theta} \frac{\bar{y}_{Q_{3}}^{\mathrm{EFT}} f_{\mathrm{TC}}}{\sqrt{M_{\mathcal{B}}^{2}+\bar{y}_{Q_{3}}^{\mathrm{EFT} 2} f_{\mathrm{TC}}^{2}}} \frac{\bar{y}_{t}^{\mathrm{EFT}} f_{\mathrm{TC}}}{\sqrt{M_{\mathcal{B}}^{2}+\bar{y}_{t}^{\mathrm{EFT} 2} f_{\mathrm{TC}}^{2}}}+\cdots,
$$

where the dots stand for higher orders in an expansion for small $s_{\theta}$. This equation should be compared to Eq. (56). We see that the two results coincide once we identify

$$
y_{Q_{3} / t} \frac{\sqrt{f_{\mathrm{TC}}}}{\sqrt{\Lambda_{\mathrm{TC}}}} \rightarrow \frac{\bar{y}_{Q_{3} / t}^{\mathrm{EFT}} f_{\mathrm{TC}}}{\sqrt{M_{\mathcal{B}}^{2}+\bar{y}_{Q_{3} / t}^{\mathrm{EFT}} f_{\mathrm{TC}}^{2}}}, \quad C_{\mathrm{Yuk}} \Lambda_{\mathrm{TC}} \rightarrow 2 M_{\mathcal{B}} .
$$

We see that the operator estimate matches if the mass of the top-partners is at its natural value $M_{\mathcal{B}} \sim \Lambda_{\mathrm{TC}}$. The mixing between SM fermions and top partners induces corrections to the gauge couplings of the top and bottom to the massive $W$ and $Z$ too, due to the fact that the top partners are vectorlike fermions [47]. In the bottom sector, we thus obtain

$$
\frac{g}{2 \cos \theta_{W}} s_{\theta}^{2} Z_{\mu} \frac{\bar{y}_{Q_{3}}^{\mathrm{EFT} 2} f_{\mathrm{TC}}^{2}}{M_{\mathcal{B}}^{2}+\bar{y}_{Q_{3}}^{\mathrm{EFT} 2} f_{\mathrm{TC}}^{2}} \bar{b}_{L} \gamma^{\mu} b_{L}+\cdots,
$$

which nicely compares with Eq. (58) once the identification in Eq. (73) is taken into account. We see, therefore, that the approach with top partners in the EFT gives the same results as the effective operators we consider, and the two actually coincide if the mass of the top partners is at the natural scale $\Lambda_{\mathrm{TC}}$. Thus, for heavy top partners, the bound from the $Z$ coupling are not problematic, as we showed in Sec. III A.

Another effective approach to partial compositeness relies on extra dimensions: it is mainly based on adapting the conjectured correspondence of anti-de Sitter (AdS) space-time with four-dimensional conformal field theories [48] to nonsupersymmetric scenarios. Models based on warped extra dimensions have been used to characterize composite Higgs bosons based on a conformal underlying theory $[49,50]$. The light Higgs boson is identified with an additional polarization of gauge fields in the bulk, thus borrowing many similarities from Gauge-Higgs unification models [51,52] (see also Ref. [53] in warped space). The mechanism of partial compositeness is described by fermions propagating in the bulk of the extra dimensions, as discussed in Refs. [54,55]. An extradimensional version of the model under study can be easily obtained by promoting the global symmetries $\mathrm{SU}\left(N_{\mathcal{F}}\right) \times \mathrm{Sp}\left(2 N_{\mathcal{S}}\right)$ to gauge symmetries in the bulk, broken by boundary conditions to the $\mathrm{SM}$ on the Planck brane, while on the $\mathrm{TeV}$ brane the breaking induced by the fermion condensate; i.e., $\mathrm{SU}\left(N_{\mathcal{F}}\right) \rightarrow \mathrm{Sp}\left(N_{\mathcal{F}}\right)$, is imposed. Composite fermions are represented by bulk fermions transforming as the bifundamental of the symmetries, while the mixing of the SM fermions, at the basis of partial compositeness, comes from explicit mass mixings on the Planck brane [56]. The theory would thus automatically describe spin-1 resonances in the form of Kaluza-Klein resonances of the gauge bosons. The advantage of extra dimensions, which is also their limitation, is the fact that the spectrum is determined by the geometry. In the model under consideration, which is not conformal in the UV, the spectrum will hardly match the prediction of a warped extra dimension.

\section{B. Pure fermionic extensions}

Traditional approaches hope to achieve partial compositeness via pure underlying gauge-fermion realisations. In this case the new composite fermion operators $\mathcal{B}$, that couple linearly to the SM fermions, must be built out of the underlying gauge-fermion dynamics. This necessarily limits its underlying composition. In addition the need to have the composite fermion operator $\mathcal{B}$ with a physical dimension such that the operator $\Psi \mathcal{B}$ (with $\Psi$ a generic SM fermion) is either superrenormalizable or marginal further constrains the underlying origin of $\mathcal{B}$. Therefore one can schematically build $\mathcal{B}$ as follows:

$\mathcal{B} \sim \mathcal{F} \mathcal{F} \mathcal{F}, \quad \mathcal{F} \mathcal{F} \mathcal{X}, \quad \mathcal{F} \mathcal{X X}, \quad \mathcal{F} \mathcal{X} \mathcal{Z}, \quad \mathcal{F} \sigma^{\mu \nu} \mathcal{G}_{\mu \nu}$,

with $\mathcal{X}$ and $\mathcal{Z}$ potentially new TC-fermions transforming according to different representations of the gauge group and $\mathcal{G}_{\mu \nu}$ the technicolor field strength. Clearly which technicolor invariant composite operator can actually be built depends on the underlying dynamics. Theories in which $\mathcal{B}$ is made by an even larger number of fermionic degrees of freedom are strongly disfavored because of the anomalously large anomalous dimensions that the composite fermion must have for $\Psi \mathcal{B}$ to be at least a marginal operator. In fact, in [57] it has been argued that even realizations with three underlying fermions are challenging. ${ }^{13}$

As noted in [11] because any purely fermionic extension [58-61] is required to have composite baryons with dimensions close to $5 / 2$, these baryons would presumably behave as if they were made by a fermion and a composite scalar similar to ours (see also [62] for a supersymmetric realisation). Naively, at some intermediate energy, our description can be viewed as an effective construction of the purely gauge-fermionic one with

$$
\mathcal{F}(\Phi) \sim \mathcal{B} \sim \mathcal{F}(\mathcal{F} \mathcal{F}), \quad \mathcal{F}(\mathcal{X} \mathcal{F}), \quad \mathcal{F}(\mathcal{X X}), \quad \mathcal{F}(\mathcal{X} \mathcal{Z})
$$

Obviously this identification is just a mnemonic, and it means that the composite baryon made by $\mathcal{F} \Phi$ can

\footnotetext{
${ }^{13}$ The remaining challenge is to build a theory that actually generates the operator $\Psi \mathcal{B}$ with the required hierarchies for the SM fermions.
} 
describe, at an intermediate effective level, one of the composite baryons with the same quantum number and physical dimensions. A similar relation can be thought for the $\mathcal{F} \sigma^{\mu \nu} \mathcal{G}_{\mu \nu}$ operator.

We can use group theory to investigate related theories. For example, from Table I of [63], we learn that model $M_{6}$, that features five two-index antisymmetric $\mathcal{F}$ under the technicolor gauge group $\mathrm{SU}(4)$ as well as three Dirac fermions in the fundamental representation $\mathcal{X}$ [61], gives rise to composite baryons $\mathcal{F} \mathcal{X X}$ and $\mathcal{F} \overline{\mathcal{X}}$. At intermediate energies these composite baryons can be mapped into a fundamental partial composite theory featuring the same $\mathcal{F}$ fermions and six two index antisymmetric TC-scalars.

\section{CONCLUSION}

We built consistent extensions of the standard model of fundamental partial composite nature and determined their electroweak effective theories in terms of the standard model fields. The bases of effective operators of different mass dimensions were built and constrained using the symmetries of the underlying theories. Our results can now be used as a stepping stone to undertake studies both in the lepton and quark flavor observables within a controlled theory of composite dynamics.

To elucidate the power of our approach, we focused on the most minimal theory of fundamental partial compositeness. We analyzed the physical consequences for the composite Higgs sector as well as the third generation quarks. Here we discovered new contributions to the Higgs potential generated from the left-handed mixing of top and bottom. Intriguingly, we also discovered that right-handed neutrinos with $\mathrm{TeV}$ scale composite Majorana masses can affect the Higgs potential with relevant consequences for the vacuum alignment of the theory. We show that constraints on the top and bottom sectors can be naturally abided. Our effective operators are ready to be deployed for full scale analyses of composite lepton and light quark flavor physics.

Finally, we provided relations with other approaches. The overall methodology can be employed to derive effective operators stemming from related underlying composite theories of dynamical electroweak symmetry breaking able to give masses to the standard model fermions.

\section{ACKNOWLEDGMENTS}

We thank C. Englert and M. Russell, and the TopFitter Collaboration, for help in providing the numerical results of their latest fits, and $\mathrm{H}$. Cai for contributing in the early stages of the project. We also acknowledge the support of the "Institut Franco-Danois" that allowed us to initiate this project. G. C. acknowledges partial support from the Labex-LIO (Lyon Institute of Origins) under Grants No. ANR-10-LABX-66 and No. FRAMA (FR3127, Fédération de Recherche "André Marie Ampère"), and thanks the CP3-Origins Institute for hospitality during the completion of this work. H. G., F. S., and A. E. T. acknowledges partial support from the Danish National Research Foundation Grant No. DNRF:90.

\section{APPENDIX A: DEFINITIONS AND NOTATION}

Whenever we write an invariant of an $\operatorname{Sp}(M)$ group, be it $\epsilon_{\mathrm{TC}}$ for $\mathrm{Sp}(2 N), \epsilon$ for $\mathrm{Sp}\left(2 N_{\mathcal{S}}\right)$, or $\varepsilon$ for $\mathrm{SU}(2)_{\mathrm{L}}$, they are defined in a similar manner. For all three $\epsilon$ 's we define

$$
\epsilon_{i j}=-\epsilon^{i j}=\left(\begin{array}{cc}
0 & -\mathbb{1} \\
\mathbb{1} & 0
\end{array}\right),
$$

where $\mathbb{1}$ is a unitary matrix or 1 depending on the group. According to usual convention we take all "up"-indices to be in the fundamental representation of a given group and "down"-indices are taken to be in the antifundamental. For the pseudoreal groups the epsilons can be used to raise or lower indices accordingly. Take e.g., the scalar field from Eq. (2) transforming in the fundamental of $\operatorname{Sp}(2 N)$

$$
\Phi^{c i}=\left(\begin{array}{c}
\mathcal{S}^{c} \\
-\epsilon_{\mathrm{TC}}^{c d} \mathcal{S}_{d}^{*}
\end{array}\right) .
$$

We note that when using the conjugate spurion field, we always use it transforming in the fundamental of $\operatorname{Sp}\left(2 N_{\mathcal{S}}\right)$, viz.

$$
\bar{\psi}^{i a}=\epsilon^{i j} \bar{\psi}_{j}^{a}=\epsilon^{i j}\left(\psi_{a}^{j}\right)^{*} .
$$

To construct the spurion field $\psi$ of the SM fermions and Yukawa couplings from the fundamental Yukawa terms, one simply embeds the TC-scalars and TC-fermions in $\mathcal{F}$ and $\Phi$ respectively. Then it is simply a matter of matching the Yukawa terms to the explicitly symmetric construction in Eq. (6). In the case of the full model presented in Sec. III C we have

$$
\mathcal{F}^{a}=\left(\begin{array}{c}
\mathcal{F}_{Q_{u}} \\
\mathcal{F}_{Q_{d}} \\
\mathcal{F}_{u} \\
\mathcal{F}_{d}
\end{array}\right), \quad \text { and } \quad \Phi^{i}=\left(\begin{array}{c}
\mathcal{S}_{q} \\
\mathcal{S}_{l} \\
-\epsilon_{\mathrm{TC}} \mathcal{S}_{q}^{*} \\
-\epsilon_{\mathrm{TC}} \mathcal{S}_{l}^{*}
\end{array}\right) \text {, }
$$

in which case one recovers the spurion field given in Eq. (69).

For the definition of the $\sigma$ matrices (and general Weylspinor algebra) we follow the notation in [64] where $\sigma^{\mu}$ and $\bar{\sigma}^{\mu}$ are defined as

$$
\sigma^{\mu}=(1, \vec{\sigma}), \quad \bar{\sigma}^{\mu}=(1,-\vec{\sigma})
$$


and $\sigma^{\mu \nu}$ is defined as

$$
\sigma^{\mu \nu}=\frac{i}{4}\left(\sigma^{\mu} \bar{\sigma}^{\nu}-\sigma^{\nu} \bar{\sigma}^{\mu}\right)
$$

\section{APPENDIX B: DETERMINING A BASIS FOR THE COMPLEX FOUR-FERMION OPERATORS}

Here we determine all possible four fermion operators respecting the symmetries of the model. The operators must be singlets under $\mathrm{SU}\left(N_{\mathcal{F}}\right), \operatorname{Sp}\left(2 N_{\mathcal{S}}\right)$, and Lorentz transformation, while being symmetric under exchange of the external fermions. The Lorentz contractions are denoted with parenthesis, $\psi^{i_{1}}{ }_{a_{1}}{ }^{\alpha} \psi^{i_{2}}{ }_{a_{2} \alpha}=\left(\psi^{i_{1}} a_{1} \psi^{i_{2}} a_{2}\right)$.

We start by noting that the operators must have the general form

$$
\mathcal{O}_{i}^{\mathrm{TC}}=\frac{1}{8 \Lambda_{\mathrm{TC}}^{2}}\left(\psi^{i_{1}}{ }_{a_{1}} \psi^{i_{2}}{ }_{a_{2}}\right)\left(\psi^{i_{3}}{ }_{a_{3}} \psi^{i_{4}}{ }_{a_{4}}\right) R_{i_{1} i_{2} i_{3} i_{4}}^{a_{1} a_{2} a_{3} a_{4}},
$$

where $R_{i_{1} i_{2} i_{3} i_{4}}^{a_{1} a_{3} a_{3}}$ is the tensor structure. This is the only kind of Lorentz structure at lowest order as any Lorentz contraction between $\sigma_{\mu}$ matrices can be written as a combination of the trivial tensors $\delta$ and $\varepsilon$. The tensor $R$ must satisfy the symmetries

$$
R_{i_{1} i_{2} i_{3} i_{4}}^{a_{1} a_{2} a_{3} a_{4}}=R_{i_{2} i_{1} i_{3} i_{4}}^{a_{2} a_{1} a_{3} a_{4}}=R_{i_{3} i_{4} i_{1} i_{2}}^{a_{3} a_{2} a_{1} a_{2}},
$$

corresponding to the exchange of the external fermions (antisymmetric parts gives vanishing contributions). On the other hand $R$ cannot be totally symmetric under exchange of all the pairs $\left(a_{s}, i_{s}\right)$. Otherwise the operator would vanish due to the fermion identity

$$
\left(f_{1} f_{2}\right)\left(f_{3} f_{4}\right)+\left(f_{1} f_{3}\right)\left(f_{2} f_{4}\right)+\left(f_{1} f_{4}\right)\left(f_{2} f_{3}\right)=0 .
$$

$R$ should furthermore be an invariant under the global symmetries $\mathrm{SU}\left(N_{\mathcal{F}}\right)$ and $\operatorname{Sp}\left(2 N_{\mathcal{S}}\right)$. For this purpose the only nontrivial tensors are the antisymmetric fermion condensate formally transforming as $\emptyset_{\mathcal{F}}$ under $\mathrm{SU}\left(N_{\mathcal{F}}\right)$, though the vacuum breaks the symmetry to $\operatorname{Sp}\left(N_{\mathcal{F}}\right)$ and the antisymmetric invariant $\epsilon$ of $\operatorname{Sp}\left(2 N_{\mathcal{S}}\right)$.

Thus one finds that $R_{i_{1} i_{2} i_{3} i_{4}}^{a_{1} a_{2} a_{3} a_{4}}$ must be a linear combination of the tensors

$$
\Sigma^{a_{\sigma(1)} a_{\sigma(2)} \Sigma^{a_{\sigma(3)}} a_{\sigma(4)}} \epsilon_{i_{\rho(1)} i_{\rho(2)}} \epsilon_{i_{\rho(3)} i_{\rho(4)}},
$$

where $\sigma, \rho$ denotes the different permutations of the integers 1 through 4 . Down to a multiplicative factor there are only nine different tensors of this type corresponding to the different ways of arranging the $a_{s}$ and $i_{s}$ indices into pairs. Constraining the tensors to satisfy the symmetry conditions of Eq. (B2) we find just five operators that span the space of the 4-fermion operators;

$$
\begin{aligned}
& \mathcal{O}_{4 f}^{6}=\frac{1}{8 \Lambda_{\mathrm{TC}}^{2}}\left(\psi^{i_{1}}{ }_{a_{1}} \psi^{i_{2}}{ }_{a_{2}}\right)\left(\psi^{i_{3}}{ }_{a_{3}} \psi^{i_{4}}{ }_{a_{4}}\right) \Sigma^{a_{1} a_{2}} \sum^{a_{3} a_{4}} \epsilon_{i_{1} i_{2}} \epsilon_{i_{3} i_{4}}, \\
& \mathcal{O}_{4 f}^{7}=\frac{1}{8 \Lambda_{\mathrm{TC}}^{2}}\left(\psi^{i_{1}}{ }_{a_{1}} \psi^{i_{2}}{ }_{a_{2}}\right)\left(\psi^{i_{3}}{ }_{a_{3}} \psi^{i_{4}}{ }_{a_{4}}\right)\left(\Sigma^{a_{1} a_{4}} \Sigma^{a_{2} a_{3}}-\Sigma^{a_{1} a_{3}} \Sigma^{a_{2} a_{4}}\right) \epsilon_{i_{1} i_{2}} \epsilon_{i_{3} i_{4}},
\end{aligned}
$$

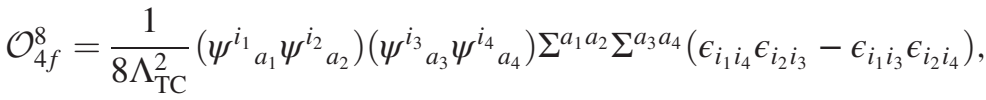

$$
\begin{aligned}
& \mathcal{O}_{4 f}^{9}=\frac{1}{8 \Lambda_{\mathrm{TC}}^{2}}\left(\psi^{i_{1}} a_{1} \psi^{i_{2}} a_{2}\right)\left(\psi^{i_{3}} a_{3} \psi^{i_{4}} a_{4}\right)\left(\Sigma^{a_{1} a_{3}} \Sigma^{a_{2} a_{4}} \epsilon_{i_{1} i_{3}} \epsilon_{i_{2} i_{4}}+\Sigma^{a_{1} a_{4}} \Sigma^{a_{2} a_{3}} \epsilon_{i_{1} i_{4}} \epsilon_{i_{2} i_{3}}\right), \\
& \mathcal{O}_{4 f}^{10}=\frac{1}{8 \Lambda_{\mathrm{TC}}^{2}}\left(\psi^{i_{1}} a_{1} \psi^{i_{2}} a_{2}\right)\left(\psi^{i_{3}} a_{3} \psi^{i_{4}} a_{4}\right)\left(\Sigma^{a_{1} a_{3}} \Sigma^{a_{2} a_{4}} \epsilon_{i_{1} i_{4}} \epsilon_{i_{2} i_{3}}+\Sigma^{a_{1} a_{4}} \Sigma^{a_{2} a_{3}} \epsilon_{i_{1} i_{3}} \epsilon_{i_{2} i_{4}}\right) .
\end{aligned}
$$

The constraint that a tensor which is totally symmetric under exchange of the pairs $\left(a_{s}, i_{s}\right)$ leads to a vanishing operators, implies the following linear dependence between the operators:

$$
\mathcal{O}_{4 f}^{6}+\mathcal{O}_{4 f}^{9}=\mathcal{O}_{4 f}^{7}+\mathcal{O}_{4 f}^{8}-\mathcal{O}_{4 f}^{10}=0 .
$$

Having used all the constraints on the operators, we find that $\mathcal{O}_{4 f}^{6}, \mathcal{O}_{4 f}^{7}$, and $\mathcal{O}_{4 f}^{8}$ make up a basis for the complex 4-fermion operators.
We note that the basis for the self-conjugate 4-fermion operators follows similarly, by noticing that any any Lorentz structure reduces to the forms $\left(\psi^{i_{1}} a_{1} \psi^{i_{2}} a_{2}\right)\left(\bar{\psi}^{i_{3} a_{3}} \bar{\psi}^{i_{4} a_{4}}\right)$.

\section{APPENDIX C: LIST OF NLO KINETIC OPERATORS}

In this appendix we list the remaining NLO operators for the chiral kinetic term, arising through loop corrections from SM fermions. All these operators contain two 
derivatives of the pNGB field and some symmetry breaking parameter(s). In the list we have ignored all the terms on the form

$$
C \operatorname{Tr}\left[\left(\Sigma^{\dagger} \stackrel{\leftrightarrow}{D}^{\mu} \Sigma\right)^{2}\right] \propto \operatorname{Tr}\left[u_{\mu} u^{\mu}\right]
$$

for some constant $C$, as these can be reabsorbed into a renormalization of the LO kinetic term. Furthermore we have utilized the fact that

$$
\operatorname{Tr}\left[\left(D_{\mu} \Sigma\right) \Sigma^{\dagger}\right]=-i \operatorname{Tr}\left[u u_{\mu} u^{\dagger}\right]=-i \operatorname{Tr} u_{\mu}=0,
$$

as the Maurer-Cartan form $u_{\mu}$ takes values in the Lie algebra of $\mathrm{SU}(4)_{\mathcal{F}}$. Any potential term containing this structure has thus been ignored.

The above consideration leave just one nontrivial, $\mathrm{SU}(4)_{\mathcal{F}}$ invariant kinetic term with only one insertion of $y_{f}^{*} y_{f}$,

$\mathcal{O}_{y \Pi D}=\frac{f_{\mathrm{TC}}^{2}}{4 \pi}\left(y_{f}^{*} y_{f}\right)^{a_{1}}{ }_{a_{2}}^{i_{1} i_{2}}\left(D_{\mu} \Sigma^{\dagger}\right)_{a_{1} a_{3}}\left(D^{\mu} \Sigma\right)^{a_{3} a_{2}} \epsilon_{i_{1} i_{2}}$.

With two insertions of $y_{f}^{*} y_{f}$ there are a total of six different contractions of the $\mathrm{SU}\left(N_{\mathcal{F}}\right)$ indices, and each of these have three different ways of contracting the $\operatorname{Sp}\left(2 N_{\mathcal{S}}\right)$ indices, only one of which is listed here (the naming is for all three operators). These operators are

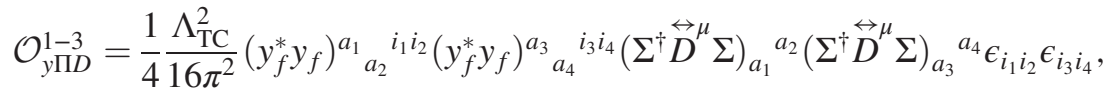

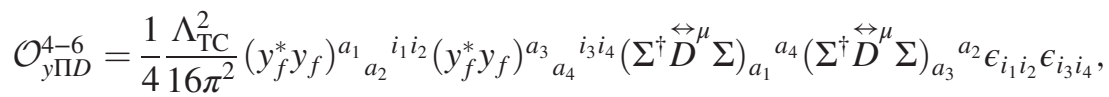

$$
\begin{aligned}
& \mathcal{O}_{y \Pi D}^{7-9}=\frac{\Lambda_{\mathrm{TC}}^{2}}{16 \pi^{2}}\left(y_{f}^{*} y_{f}\right)^{a_{1}} a_{2} i_{1} i_{2}\left(y_{f}^{*} y_{f}\right)^{a_{3}} a_{4} i_{3} i_{4}\left(D_{\mu} \Sigma^{\dagger}\right)_{a_{1} a_{3}}\left(D^{\mu} \Sigma\right)^{a_{2} a_{4}} \epsilon_{i_{1} i_{2}} \epsilon_{i_{3} i_{4}} \\
& \mathcal{O}_{y \Pi D}^{10-12}=\frac{\Lambda_{\mathrm{TC}}^{2}}{16 \pi^{2}}\left(y_{f}^{*} y_{f}\right)^{a_{1}}{ }_{a_{1}}{ }^{i_{1} i_{2}}\left(y_{f}^{*} y_{f}\right)^{a_{2}}{ }_{a_{3}}^{i_{3} i_{4}}\left(D_{\mu} \Sigma^{\dagger}\right)_{a_{2} a_{4}}\left(D^{\mu} \Sigma\right)^{a_{4} a_{3}} \epsilon_{i_{1} i_{2}} \epsilon_{i_{3} i_{4}}, \\
& \mathcal{O}_{y \Pi D}^{13-15}=\frac{\Lambda_{\mathrm{TC}}^{2}}{16 \pi^{2}}\left(y_{f}^{*} y_{f}\right)^{a_{1}}{ }_{a_{2}}^{i_{1} i_{2}}\left(y_{f}^{*} y_{f}\right)^{a_{2}} a_{3} i_{3} i_{4}\left(D_{\mu} \Sigma^{\dagger}\right)_{a_{1} a_{4}}\left(D^{\mu} \Sigma\right)^{a_{4} a_{3}} \epsilon_{i_{1} i_{2}} \epsilon_{i_{3} i_{4}}, \\
& \mathcal{O}_{y \Pi D}^{16-18}=\frac{1}{2} \frac{\Lambda_{\mathrm{TC}}^{2}}{16 \pi^{2}}\left(y_{f}^{*} y_{f}\right)^{a_{1}}{ }_{a_{2}}{ }^{i_{1} i_{2}}\left(y_{f}^{*} y_{f}\right)^{a_{3}} a_{4}{ }_{a_{3} i_{4}} \sum_{a_{1} a_{3}}^{\dagger}\left(D^{\mu} \Sigma\right)^{a_{2} a_{5}}\left(\Sigma^{\dagger} \stackrel{\leftrightarrow}{D} \Sigma\right)_{a_{5}}{ }^{a_{4}} \epsilon_{i_{1} i_{2}} \epsilon_{i_{3} i_{4}},
\end{aligned}
$$

where the last operator is complex.

There are four real operators with two EW gauge insertion

$$
\begin{aligned}
& \mathcal{O}_{\Pi D}^{1}=\frac{1}{4} \frac{f_{\mathrm{TC}}^{2}}{16 \pi^{2}} \operatorname{Tr}\left[\left(\Sigma \stackrel{\leftrightarrow}{D}_{\mu} \Sigma^{\dagger}\right) T_{\mathcal{F}}^{I}\left(\Sigma \stackrel{\leftrightarrow}{D}^{\mu} \Sigma^{\dagger}\right) T_{\mathcal{F}}^{I}\right] \\
& \mathcal{O}_{\Pi D}^{2}=\frac{1}{4} \frac{f_{\mathrm{TC}}^{2}}{16 \pi^{2}} \operatorname{Tr}\left[\left(\Sigma \stackrel{\leftrightarrow}{D}_{\mu} \Sigma^{\dagger}\right) T_{\mathcal{F}}^{I}\right] \operatorname{Tr}\left[\left(\Sigma \stackrel{\leftrightarrow}{D}^{\mu} \Sigma^{\dagger}\right) T_{\mathcal{F}}^{I}\right] \\
& \mathcal{O}_{\Pi D}^{3}=\frac{f_{\mathrm{TC}}^{2}}{16 \pi^{2}} \operatorname{Tr}\left[\left(D_{\mu} \Sigma\right)\left(D^{\mu} \Sigma\right)^{\dagger} T_{\mathcal{F}}^{I} T_{\mathcal{F}}^{I}\right] \\
& \mathcal{O}_{\Pi D}^{4}=\frac{f_{\mathrm{TC}}^{2}}{16 \pi^{2}} \operatorname{Tr}\left[\left(D_{\mu} \Sigma\right)\left(T_{\mathcal{F}}^{I}\right)^{\mathrm{T}}\left(D^{\mu} \Sigma\right)^{\dagger} T_{\mathcal{F}}^{I}\right]
\end{aligned}
$$

where the trace is over the $\mathrm{SU}\left(N_{\mathcal{F}}\right)$ indices. Additionally there is 1 complex operator too,

$$
\mathcal{O}_{\Pi D}^{5}=\frac{1}{2} \frac{f_{\mathrm{TC}}^{2}}{16 \pi^{2}} \operatorname{Tr}\left[\left(D_{\mu} \Sigma\right)\left(\Sigma^{\dagger} \stackrel{\stackrel{\leftrightarrow}{D}}{\mu} \Sigma\right)\left(T_{\mathcal{F}}^{I}\right)^{\mathrm{T}} \Sigma^{\dagger} T_{\mathcal{F}}^{I}\right]
$$

Finally there is one complex term involving the fundamental fermion mass,

$$
\mathcal{O}_{m \Pi D}=\frac{1}{2} \frac{f_{\mathrm{TC}}^{2}}{16 \pi^{2}} \operatorname{Tr}\left[\left(D_{\mu} \Sigma\right) \chi^{*}\left(\Sigma \stackrel{\leftrightarrow}{D}^{\mu} \Sigma^{\dagger}\right)\right]
$$

\section{APPENDIX D: LIST OF FOUR-FERMION OPERATORS}

We now list all the four-fermion operators found in the model containing only top and bottom SM fermions. These are found by expanding the operators $\mathcal{O}_{4 f}^{1, \ldots, 8}$. As it is usually done color indices are always contracted along the spinor structure, and where needed we have made use of the $\mathrm{SU}(3)_{c}$ generators $T^{A}=\frac{1}{2} \lambda^{A}$. 
Operators with four left-handed quarks,

$$
\begin{aligned}
\mathcal{L}_{\mathrm{EFT}} \supset & \frac{C_{4 f}^{4}+C_{4 f}^{5}}{4} \frac{\left|y_{Q_{3}}\right|^{4}}{\Lambda_{\mathrm{TC}}^{2}}\left[\left(\bar{t}_{\mathrm{L}} \gamma_{\mu} t_{\mathrm{L}}\right)\left(\bar{t}_{\mathrm{L}} \gamma^{\mu} t_{\mathrm{L}}\right)+\left(\bar{b}_{\mathrm{L}} \gamma_{\mu} b_{\mathrm{L}}\right)\left(\bar{b}_{\mathrm{L}} \gamma^{\mu} b_{\mathrm{L}}\right)\right]+\frac{c_{\theta}^{2} C_{4 f}^{3}+C_{4 f}^{4}}{2} \frac{\left|y_{Q_{3}}\right|^{4}}{\Lambda_{\mathrm{TC}}^{2}}\left(\bar{b}_{\mathrm{L}} \gamma_{\mu} b_{\mathrm{L}}\right)\left(\bar{t}_{\mathrm{L}} \gamma^{\mu} t_{\mathrm{L}}\right) \\
& +\frac{-c_{\theta}^{2} C_{4 f}^{3}+C_{4 f}^{5}}{2} \frac{\left|y_{Q_{3}}\right|^{4}}{\Lambda_{\mathrm{TC}}^{2}}\left(\bar{b}_{\mathrm{L}} \gamma_{\mu} t_{\mathrm{L}}\right)\left(\bar{t}_{\mathrm{L}} \gamma^{\mu} b_{\mathrm{L}}\right) .
\end{aligned}
$$

Operators with four right-handed quarks,

$$
\begin{aligned}
\mathcal{L}_{\mathrm{EFT}} \supset & \frac{C_{4 f}^{4}+C_{4 f}^{5}}{4} \frac{\left|y_{t}\right|^{4}}{\Lambda_{\mathrm{TC}}^{2}}\left(\bar{t}_{\mathrm{R}} \gamma_{\mu} t_{\mathrm{R}}\right)\left(\bar{t}_{\mathrm{R}} \gamma^{\mu} t_{\mathrm{R}}\right)+\frac{C_{4 f}^{4}+C_{4 f}^{5}}{4} \frac{\left|y_{b}\right|^{4}}{\Lambda_{\mathrm{TC}}^{2}}\left(\bar{b}_{\mathrm{R}} \gamma_{\mu} b_{\mathrm{R}}\right)\left(\bar{b}_{\mathrm{R}} \gamma^{\mu} b_{\mathrm{R}}\right) \\
& +\frac{c_{\theta}^{2} C_{4 f}^{3}+C_{4 f}^{4}}{2} \frac{\left|y_{t} y_{b}\right|^{2}}{\Lambda_{\mathrm{TC}}^{2}}\left(\bar{b}_{\mathrm{R}} \gamma_{\mu} b_{\mathrm{R}}\right)\left(\bar{t}_{\mathrm{R}} \gamma^{\mu} t_{\mathrm{R}}\right)+\frac{-c_{\theta}^{2} C_{4 f}^{3}+C_{4 f}^{5}}{2} \frac{\left|y_{t} y_{b}\right|^{2}}{\Lambda_{\mathrm{TC}}^{2}}\left(\bar{b}_{\mathrm{R}} \gamma_{\mu} t_{\mathrm{R}}\right)\left(\bar{t}_{\mathrm{R}} \gamma^{\mu} b_{\mathrm{R}}\right) .
\end{aligned}
$$

Operators with two left-handed and two right-handed top quarks,

$$
\begin{aligned}
\mathcal{L}_{\mathrm{EFT}} \supset & \left(-s_{\theta}^{2} C_{4 f}^{1}+C_{4 f}^{2}\right) \frac{\left|y_{Q_{3}} y_{t}\right|^{2}}{\Lambda_{\mathrm{TC}}^{2}}\left(\bar{t}_{\mathrm{R}} t_{\mathrm{L}}\right)\left(\bar{t}_{\mathrm{L}} t_{\mathrm{R}}\right)-\frac{s_{\theta}^{2} C_{4 f}^{3}+C_{4 f}^{4}}{2} \frac{\left|y_{Q_{3}} y_{t}\right|^{2}}{\Lambda_{\mathrm{TC}}^{2}}\left(\bar{t}_{\mathrm{L}} \gamma_{\mu} t_{\mathrm{L}}\right)\left(\bar{t}_{\mathrm{R}} \gamma^{\mu} t_{\mathrm{R}}\right) \\
& +\left(\frac{s_{\theta}^{2} y_{Q_{3}}^{2} y_{t}^{2}}{\Lambda_{\mathrm{TC}}^{2}}\left[\frac{3 C_{4 f}^{6}-3 C_{4 f}^{7}-C_{4 f}^{8}}{6}\left(\bar{t}_{\mathrm{R}} t_{\mathrm{L}}\right)\left(\bar{t}_{\mathrm{R}} t_{\mathrm{L}}\right)-C_{4 f}^{8}\left(\bar{t}_{\mathrm{R}} T^{A} t_{\mathrm{L}}\right)\left(\bar{t}_{\mathrm{R}} T^{A} t_{\mathrm{L}}\right)\right]+\text { H.c. }\right) .
\end{aligned}
$$

Operators with two left-handed and two right-handed bottom quarks:

$$
\begin{aligned}
& \mathcal{L}_{\mathrm{EFT}} \supset\left(-s_{\theta}^{2} C_{4 f}^{1}+C_{4 f}^{2}\right) \frac{\left|y_{Q_{3}} y_{b}\right|^{2}}{\Lambda_{\mathrm{TC}}^{2}}\left(\bar{b}_{\mathrm{R}} b_{\mathrm{L}}\right)\left(\bar{b}_{\mathrm{L}} b_{\mathrm{R}}\right)-\frac{s_{\theta}^{2} C_{4 f}^{3}+C_{4 f}^{4}}{2} \frac{\left|y_{Q_{3}} y_{b}\right|^{2}}{\Lambda_{\mathrm{TC}}^{2}}\left(\bar{b}_{\mathrm{L}} \gamma_{\mu} b_{\mathrm{L}}\right)\left(\bar{b}_{\mathrm{R}} \gamma^{\mu} b_{\mathrm{R}}\right) \\
&+\left(\frac{s_{\theta}^{2} y_{Q_{3}}^{2} y_{b}^{2}}{\Lambda_{\mathrm{TC}}^{2}}\left[\frac{3 C_{4 f}^{6}-3 C_{4 f}^{7}-C_{4 f}^{8}}{6}\left(\bar{b}_{\mathrm{R}} b_{\mathrm{L}}\right)\left(\bar{b}_{\mathrm{R}} b_{\mathrm{L}}\right)-C_{4 f}^{8}\left(\bar{b}_{\mathrm{R}} T^{A} b_{\mathrm{L}}\right)\left(\bar{b}_{\mathrm{R}} T^{A} b_{\mathrm{L}}\right)\right]+\text { H.c. }\right) .
\end{aligned}
$$

Operators with two left-handed and two right-handed quarks, either top and bottom respectively or vice versa,

$$
\begin{aligned}
\mathcal{L}_{\mathrm{EFT}} \supset & C_{4 f}^{2} \frac{\left|y_{Q_{3}}\right|^{2}}{\Lambda_{\mathrm{TC}}^{2}}\left[\left|y_{t}\right|^{2}\left(\bar{t}_{\mathrm{R}} b_{\mathrm{L}}\right)\left(\bar{b}_{\mathrm{L}} t_{\mathrm{R}}\right)+\left|y_{b}\right|^{2}\left(\bar{t}_{\mathrm{L}} b_{\mathrm{R}}\right)\left(\bar{b}_{\mathrm{R}} t_{\mathrm{L}}\right)\right] \\
& -\frac{C_{4 f}^{4}}{2} \frac{\left|y_{Q_{3}}\right|^{2}}{\Lambda_{\mathrm{TC}}^{2}}\left[\left|y_{t}\right|^{2}\left(\bar{b}_{\mathrm{L}} \gamma_{\mu} b_{\mathrm{L}}\right)\left(\bar{t}_{\mathrm{R}} \gamma^{\mu} t_{\mathrm{R}}\right)+\left|y_{b}\right|^{2}\left(\bar{t}_{\mathrm{L}} \gamma_{\mu} t_{\mathrm{L}}\right)\left(\bar{b}_{\mathrm{R}} \gamma^{\mu} b_{\mathrm{R}}\right)\right] .
\end{aligned}
$$

Operators with a left-handed and right-handed top quark, and a left-handed and right-handed bottom quark,

$$
\begin{aligned}
\mathcal{L}_{\mathrm{EFT}} \supset- & C_{4 f}^{1} \frac{s_{\theta}^{2}\left|y_{Q_{3}}\right|^{2}}{\Lambda_{\mathrm{TC}}^{2}}\left[y_{t} y_{b}^{*}\left(\bar{t}_{\mathrm{R}} t_{\mathrm{L}}\right)\left(\bar{b}_{\mathrm{L}} b_{\mathrm{R}}\right)+y_{b} y_{t}^{*}\left(\bar{b}_{\mathrm{R}} b_{\mathrm{L}}\right)\left(\bar{t}_{\mathrm{L}} t_{\mathrm{R}}\right)\right]-\frac{C_{4 f}^{3}}{2} \frac{s_{\theta}^{2}\left|y_{Q_{3}}\right|^{2}}{\Lambda_{\mathrm{TC}}^{2}}\left[y_{t} y_{b}^{*}\left(\bar{b}_{\mathrm{L}} \gamma_{\mu} t_{\mathrm{L}}\right)\left(\bar{t}_{\mathrm{R}} \gamma^{\mu} b_{\mathrm{R}}\right)+y_{b} y_{t}^{*}\left(\bar{t}_{\mathrm{L}} \gamma_{\mu} b_{\mathrm{L}}\right)\left(\bar{b}_{\mathrm{R}} \gamma^{\mu} t_{\mathrm{R}}\right)\right] \\
+ & \left(2 C_{4 f}^{8} \frac{y_{Q_{3}}^{2} y_{t} y_{b}}{\Lambda_{\mathrm{TC}}^{2}}\left[c_{2 \theta}\left(\bar{b}_{\mathrm{R}} T^{A} b_{\mathrm{L}}\right)\left(\bar{t}_{\mathrm{R}} T^{A} t_{\mathrm{L}}\right)-c_{\theta}^{2}\left(\bar{b}_{\mathrm{R}} T^{A} t_{\mathrm{L}}\right)\left(\bar{t}_{\mathrm{R}} T^{A} b_{\mathrm{L}}\right)\right]+\frac{-3 C_{4 f}^{7}+2 c_{\theta}^{2} C_{4 f}^{8} \frac{y_{Q_{3}}^{2} y_{t} y_{b}}{\Lambda_{\mathrm{TC}}^{2}}\left(\bar{b}_{\mathrm{R}} t_{\mathrm{L}}\right)\left(\bar{t}_{\mathrm{R}} b_{\mathrm{L}}\right)}{3} \frac{\left.3 s_{\theta}^{2} C_{4 f}^{6}+3 c_{\theta}^{2} C_{4 f}^{7}-\left(1+c_{\theta}^{2}\right) C_{4 f}^{8} \frac{y_{Q_{3}}^{2} y_{t} y_{b}}{\Lambda_{\mathrm{TC}}^{2}}\left(\bar{b}_{\mathrm{R}} b_{\mathrm{L}}\right)\left(\bar{t}_{\mathrm{R}} t_{\mathrm{L}}\right)+\text { H.c. }\right)}{3}\right.
\end{aligned}
$$


[1] S. Weinberg, Phys. Rev. D 13, 974 (1976).

[2] S. Dimopoulos and L. Susskind, Nucl. Phys. B155, 237 (1979).

[3] E. Eichten and K. D. Lane, Phys. Lett. 90B, 125 (1980).

[4] E. H. Simmons, Nucl. Phys. B312, 253 (1989).

[5] S. Samuel, Nucl. Phys. B347, 625 (1990).

[6] A. Kagan and S. Samuel, Phys. Lett. B 270, 37 (1991).

[7] C. D. Carone and E. H. Simmons, Nucl. Phys. B397, 591 (1993).

[8] C. D. Carone, E. H. Simmons, and Y. Su, Phys. Lett. B 344, 287 (1995).

[9] M. Antola, M. Heikinheimo, F. Sannino, and K. Tuominen, J. High Energy Phys. 03 (2010) 050.

[10] D. B. Kaplan, Nucl. Phys. B365, 259 (1991).

[11] F. Sannino, A. Strumia, A. Tesi, and E. Vigiani, J. High Energy Phys. 11 (2016) 029.

[12] D. B. Kaplan and H. Georgi, Phys. Lett. 136B, 183 (1984).

[13] A. Kagan, in 15th Johns Hopkins Workshop on Current Problems in Particle Theory: Particle Physics from Underground to Heaven Baltimore, Maryland, 1991 (World Scientific Publishing, Singapore, 1991), p. 217.

[14] B. A. Dobrescu, Nucl. Phys. B449, 462 (1995).

[15] A. L. Kagan, Phys. Rev. D 51, 6196 (1995).

[16] M. Antola, S. Di Chiara, F. Sannino, and K. Tuominen, Eur. Phys. J. C 71, 1784 (2011).

[17] M. Antola, S. Di Chiara, F. Sannino, and K. Tuominen, Nucl. Phys. B864, 664 (2012).

[18] W. Altmannshofer, S. Gori, A. L. Kagan, L. Silvestrini, and J. Zupan, Phys. Rev. D 93, 031301 (2016).

[19] G. Cacciapaglia and F. Sannino, J. High Energy Phys. 04 (2014) 111.

[20] R. Lewis, C. Pica, and F. Sannino, Phys. Rev. D 85, 014504 (2012).

[21] G. Buchalla, O. Catá, and C. Krause, Phys. Lett. B 731, 80 (2014).

[22] H. Georgi, Phys. Lett. B 298, 187 (1993).

[23] S. R. Coleman, J. Wess, and B. Zumino, Phys. Rev. 177, 2239 (1969).

[24] C. G. Callan, Jr., S. R. Coleman, J. Wess, and B. Zumino, Phys. Rev. 177, 2247 (1969).

[25] J. Bijnens and J. Lu, J. High Energy Phys. 11 (2009) 116.

[26] J. Bijnens and J. Lu, J. High Energy Phys. 03 (2011) 028.

[27] M. Hansen, K. Langæble, and F. Sannino, Phys. Rev. D 92 , 075036 (2015).

[28] Z.-y. Duan, P. S. Rodrigues da Silva, and F. Sannino, Nucl. Phys. B592, 371 (2001).

[29] B. Grzadkowski, M. Iskrzynski, M. Misiak, and J. Rosiek, J. High Energy Phys. 10 (2010) 085.

[30] T. A. Ryttov and F. Sannino, Phys. Rev. D 78, 115010 (2008).

[31] J. Galloway, J. A. Evans, M. A. Luty, and R. A. Tacchi, J. High Energy Phys. 10 (2010) 086.

[32] M. J. Dugan, H. Georgi, and D. B. Kaplan, Nucl. Phys. B254, 299 (1985).

[33] E. Katz, A. E. Nelson, and D. G. E. Walker, J. High Energy Phys. 08 (2005) 074.

[34] M. Baak, J. Cúth, J. Haller, A. Hoecker, R. Kogler, K. Mönig, M. Schott, and J. Stelzer (Gfitter Group), Eur. Phys. J. C 74, 3046 (2014).
[35] S. Gori, J. Gu, and L.-T. Wang, J. High Energy Phys. 04 (2016) 062.

[36] S. Schael et al. (SLD Electroweak Group, DELPHI, ALEPH, SLD, SLD Heavy Flavour Group, OPAL, LEP Electroweak Working Group, L3), Phys. Rep. 427, 257 (2006).

[37] A. Arbey, G. Cacciapaglia, H. Cai, A. Deandrea, S. Le Corre, and F. Sannino, Phys. Rev. D 95, 015028 (2017).

[38] M. Tanabashi, K. Yamawaki, and K.-I. Kondo, in Dynamical symmetry breaking. Proceedings, Workshop, Nagoya, Japan, 1989, Univ., Dept. Phys. (Univ., Dept. Phys., Nagoya, Japan, 1990), p. 28.

[39] K.-i. Kondo, A. Shibata, M. Tanabashi, and K. Yamawaki, Prog. Theor. Phys. 91, 541 (1994); 93, 489(E) (1995).

[40] T.A. Collaboration (ATLAS), Report No. ATLAS-CONF2016-104.

[41] G. Durieux, F. Maltoni, and C. Zhang, Phys. Rev. D 91, 074017 (2015).

[42] A. Buckley, C. Englert, J. Ferrando, D. J. Miller, L. Moore, M. Russell, and C. D. White, J. High Energy Phys. 04 (2016) 015.

[43] D. Marzocca, M. Serone, and J. Shu, J. High Energy Phys. 08 (2012) 013.

[44] G. Panico and A. Wulzer, Lect. Notes Phys. 913, 1 (2016).

[45] M. Redi and A. Tesi, J. High Energy Phys. 10 (2012) 166.

[46] A. Pomarol and F. Riva, J. High Energy Phys. 08 (2012) 135.

[47] F. del Aguila, M. Perez-Victoria, and J. Santiago, J. High Energy Phys. 09 (2000) 011.

[48] J. M. Maldacena, Int. J. Theor. Phys. 38, 1113 (1999); Adv. Theor. Math. Phys. 2, 231 (1998).

[49] R. Contino, Y. Nomura, and A. Pomarol, Nucl. Phys. B671, 148 (2003).

[50] K. Agashe, R. Contino, and A. Pomarol, Nucl. Phys. B719, 165 (2005).

[51] Y. Hosotani, Phys. Lett. 126B, 309 (1983).

[52] I. Antoniadis, K. Benakli, and M. Quiros, New J. Phys. 3, 20 (2001).

[53] Y. Hosotani and M. Mabe, Phys. Lett. B 615, 257 (2005).

[54] R. Contino and A. Pomarol, J. High Energy Phys. 11 (2004) 058.

[55] G. Cacciapaglia, G. Marandella, and J. Terning, J. High Energy Phys. 06 (2009) 027.

[56] C. A. Scrucca, M. Serone, and L. Silvestrini, Nucl. Phys. B669, 128 (2003).

[57] C. Pica and F. Sannino, Phys. Rev. D 94, 071702 (2016).

[58] J. Barnard, T. Gherghetta, and T. S. Ray, J. High Energy Phys. 02 (2014) 002.

[59] G. Ferretti and D. Karateev, J. High Energy Phys. 03 (2014) 077.

[60] L. Vecchi, J. High Energy Phys. 02 (2017) 094.

[61] G. Ferretti, J. High Energy Phys. 06 (2014) 142.

[62] F. Caracciolo, A. Parolini, and M. Serone, J. High Energy Phys. 02 (2013) 066.

[63] A. Belyaev, G. Cacciapaglia, H. Cai, G. Ferretti, T. Flacke, A. Parolini, and H. Serodio, J. High Energy Phys. 01 (2017) 094; 12 (2017) 088(E).

[64] H. K. Dreiner, H. E. Haber, and S. P. Martin, Phys. Rep. 494, 1 (2010). 